\title{
1 Structure and allosteric regulation of human IDH3 holoenzyme
}

\section{Pengkai Sun ${ }^{1}$, Yan Liu ${ }^{2}$, Tengfei Ma ${ }^{1}$, and Jianping Ding ${ }^{1,2,3, *}$}

$3{ }^{1}$ State Key Laboratory of Molecular Biology, Shanghai Institute of Biochemistry and Cell 4 Biology, Center for Excellence in Molecular Cell Science, University of Chinese Academy of 5 Sciences, Chinese Academy of Sciences, 320 Yueyang Road, Shanghai 200031, China

$6{ }^{2}$ School of Life Science and Technology, ShanghaiTech University, 393 Huaxia Zhong Road,

7 Shanghai 201210, China

$8{ }^{3}$ School of Life Science, Hangzhou Institute for Advanced Study, University of Chinese

9 Academy of Sciences, 1 Xiangshan Road, Hangzhou 310024, China

10

$11 *$ * Correspondence: Jianping Ding (Phone: +86-21-54921619, E-mail: jpding@sibcb.ac.cn).

12 Running title: Crystal structure of human NAD-IDH

13 Keywords: Isocitrate dehydrogenase, IDH, NAD-IDH, assembly, allosteric regulation. 
15 Abstract Human NAD-dependent isocitrate dehydrogenase or IDH3 catalyzes the 16 decarboxylation of isocitrate into $\alpha$-ketoglutarate in the TCA cycle. We here report the 17 structure of the IDH3 holoenzyme, in which the $\alpha \beta$ and $\alpha \gamma$ heterodimers assemble the $\alpha_{2} \beta \gamma$ 18 heterotetramer via their clasp domains, and two $\alpha_{2} \beta \gamma$ heterotetramers assemble the $\left(\alpha_{2} \beta \gamma\right)_{2}$ 19 heterooctamer via the $\beta$ and $\gamma$ subunits. The functional roles of the key residues involved in 20 the assembly and allosteric regulation are validated by mutagenesis and kinetic studies. The 21 allosteric site plays an important role but the pseudo allosteric site plays no role in the 22 allosteric activation; the activation signal from the allosteric site is transmitted to the active 23 sites of both heterodimers via the clasp domains; and the N-terminus of the $\gamma$ subunit plays a 24 critical role in the formation and function of the holoenzyme. These findings reveal the 25 molecular mechanism of the assembly and allosteric regulation of human IDH3 holoenzyme. 


\section{Introduction}

In all aerobic organisms, the cells use the tricarboxylic acid (TCA) cycle (also called citric acid cycle or Krebs cycle) to generate ATP through oxidation of acetyl-CoA derived from carbohydrates, fats, and proteins. In addition, the TCA cycle also provides intermediates for de novo synthesis of proteins, lipids and nucleic acids (Pavlova and Thompson, 2016). Among a series of biochemical reactions in the TCA cycle, isocitrate dehydrogenases (IDHs) catalyze oxidative decarboxylation of isocitrate (ICT) into $\alpha$-ketoglutarate $(\alpha-K G)$ using NAD or NADP as coenzyme. Most prokaryotes contain only NADP-dependent IDHs (NADP-IDHs) in the cytosol, which exert the catalytic function. Eukaryotes contain both NADP-IDHs and NAD-dependent IDHs (NAD-IDHs). In human and other mammalian cells, there are two NADP-IDHs and one NAD-IDH: the two NADP-IDHs are located to the cytosol and the mitochondria and are called IDH1 and IDH2, respectively, and the NAD-IDH is located to the mitochondria and is called IDH3. It is well established that IDH3 exerts the catalytic function in the TCA cycle (Al-Khallaf, 2017). Both IDH1 and IDH2 play important roles in cellular defense against oxidative damage, removal of reactive oxygen species, and synthesis of fat and cholesterol (Jo et al., 2001; Kim and Park, 2003; Koh et al., 2004; Lee et al., 2002). Aberrant functions of all three enzymes have been implicated in the pathogenesis of numerous metabolic diseases (Hartong et al., 2008; Kiefmann et al., 2017; Yoshimi et al., 2016) and malignant tumors (Dang et al., 2009; May et al., 2019; Waitkus et al., 2016; Yan et al., 2009; Zhang et al., 2015).

Both prokaryotic and eukaryotic NADP-IDHs exist and function as homodimers in which both subunits have catalytic activity (Hurley et al., 1991; Xu et al., 2004). These enzymes share a conserved catalytic mechanism, but have different regulatory mechanisms. The activity of Escherichia coli NADP-IDH is regulated through reversible phosphorylation and dephosphorylation of a strictly conserved Ser residue at the active site by the dual functional kinase/phosphatase AceK, and other bacterial NADP-IDHs might share a similar regulatory mechanism (Zheng and Jia, 2010; Zheng et al., 2012). The activity of human IDH1 is regulated through substrate-binding induced conformational change of a key structure element at the active site, and other mammalian NADP-IDHs might utilize a similar regulatory mechanism (Xu et al., 2004; Yang et al., 2010).

Compared to NADP-IDHs, NAD-IDHs are composed of different types of subunits with distinct functions and employ more sophisticated regulatory mechanisms. Saccharomyces cerevisiae NAD-IDH is composed of a regulatory subunit IDH1 and a catalytic subunit IDH2 which form the IDH1/IDH2 heterodimer as the basic functional unit, and the heterodimer is assembled into a heterotetramer and further into a heterooctamer (Lin et al., 2011; Lin and McAlister-Henn, 2003; Taylor et al., 2008). IDH2 contains the active site and IDH1 contains 
the allosteric site, and the binding of activators citrate (CIT) and AMP to the allosteric site can cause conformational changes of the active site through the heterodimer interface, leading to the activation of the enzyme. The composition and regulation of human and other mammalian NAD-IDHs are even more complex than those of yeast NAD-IDH. Human NAD-IDH or IDH3 are composed of three types of subunits in the ratio of $2 \alpha: 1 \beta: 1 \gamma$ (Nichols et al., 1993; Nichols et al., 1995). The $\alpha$ and $\beta$ subunits form a heterodimer $(\alpha \beta)$ and the $\alpha$ and $\gamma$ subunits form another heterodimer $(\alpha \gamma)$, and the two heterodimers are assembled into the $\alpha_{2} \beta \gamma$ heterotetramer and further into the $\left(\alpha_{2} \beta \gamma\right)_{2}$ heterooctamer (also called holoenzyme) (Ehrlich and Colman, 1983; Ehrlich et al., 1981).

Early biochemical studies of mammalian NAD-IDHs showed that the $\alpha$ subunit is the catalytic subunit, and the $\beta$ and $\gamma$ subunits are the regulatory subunits (Cohen and Colman, 1972; Ehrlich and Colman, 1981); and the activity of the holoenzyme is positively regulated by CIT and ADP but negatively regulated by ATP and NADH (Gabriel and Plaut, 1984a; Gabriel et al., 1985; Gabriel and Plaut, 1984b). Our biochemical studies of human IDH3 confirmed some results from the previous studies but also revealed some new findings. We found that in the IDH3 holoenzyme, the $\alpha$ subunits of both $\alpha \beta$ and $\alpha \gamma$ heterodimers have catalytic activity; however, only the $\gamma$ subunit plays a regulatory role via an allosteric regulatory mechanism, while the $\beta$ subunit plays no regulatory role but is required for the function of the holoenzyme (Ma et al., 2017b). The holoenzyme and the $\alpha \gamma$ heterodimer are positively regulated by CIT and ADP, and negatively regulated by NADH. In addition, these enzymes can be activated by low concentrations of ATP, but inhibited by high concentrations of ATP. In contrast, the $\alpha \beta$ heterodimer cannot be activated by CIT and ADP, and is inhibited by both NADH and ATP. Our detailed structural and biochemical studies of the $\alpha \gamma$ and $\alpha \beta$ heterodimers revealed the underlying molecular mechanisms (Liu et al., 2018; Ma et al., 2017a; Sun et al., 2020; Sun et al., 2019). Nevertheless, so far the structure, assembly and regulatory mechanism of human IDH3 holoenzyme are still unknown. Thus, how the $\alpha \beta$ and $\alpha \gamma$ heterodimers are assembled into the $\alpha_{2} \beta \gamma$ heterotetramer and further into the $\left(\alpha_{2} \beta \gamma\right)_{2}$ heterooctamer is unclear. How the allosteric site in the $\gamma$ subunit regulates both $\alpha$ subunits in the $\alpha_{2} \beta \gamma$ heterotetramer is also unclear. In addition, whether the regulatory mechanisms of the $\alpha \beta$ and $\alpha \gamma$ heterodimers are applicable to the holoenzyme remains elusive.

In this work, we determined the crystal structure of human IDH3 holoenzyme in apo form. In the holoenzyme, the $\alpha \beta$ and $\alpha \gamma$ heterodimers assemble the $\alpha_{2} \beta \gamma$ heterotetramer via their clasp domains, and two $\alpha_{2} \beta \gamma$ heterotetramers assemble the $\left(\alpha_{2} \beta \gamma\right)_{2}$ heterooctamer through the N-terminus of the $\gamma$ subunit of one heterotetramer inserting into the back cleft of the $\beta$ subunit of the other heterotetramer. We further performed mutagenesis and kinetic 
studies to validate the functional roles of the key residues at the allosteric site, the pseudo allosteric site, the heterodimer interface, and the heterodimer-heterodimer interface, as well as the N-terminus of the $\gamma$ subunit. Our structural and biochemical data together reveal the molecular mechanism for the assembly and allosteric regulation of human IDH3 holoenzyme.

\section{Results}

\section{Preparation and biochemical analysis of human IDH3 holoenzyme}

The wild-type human IDH3 holoenzyme was prepared as described previously (Ma et al., 2017b). Crystallization of the wild-type IDH3 holoenzyme yielded crystals at various conditions; however, these crystals diffracted X-rays to low resolution (about $10 \AA$ ), prohibiting us from determining the crystal structure. Our previous biochemical and structural studies showed that substitution of the C-terminal region of the $\beta$ subunit (residues 341-349) with that of the $\alpha$ subunit (residues 330-338) produced a stable $\alpha \beta$ mutant which exhibits similar enzymatic properties as the wild-type enzyme, and this $\alpha \beta$ mutant yielded high quality crystals which allowed us to solve the structure of the $\alpha \beta$ heterodimer (Sun et al., 2019). Thus, we prepared a mutant IDH3 holoenzyme containing the $\beta$ mutant, which led to successful structure determination of the IDH3 holoenzyme.

Like the wild-type holoenzyme, the $\beta$ mutant holoenzyme exists as a heterooctamer in solution with high purity and homogeneity as shown by SDS-PAGE and size exclusion chromatography (SEC) analyses (Fig. S1). The $\beta$ mutant holoenzyme exhibits almost identical enzymatic properties as the wild-type holoenzyme, indicating that the substitution of the C-terminal region of the $\beta$ subunit has no effects on the biochemical and enzymatic properties of the holoenzyme (Fig. S2 and Table S1). Therefore, we will not distinguish the wild-type and the $\beta$ mutant holoenzyme hereafter.

\section{Structure of human IDH3 holoenzyme}

The structure of human IDH3 holoenzyme was solved at $3.47 \AA$ resolution (Table 1). The crystals of the IDH3 holoenzyme belong to space group $I 4_{1} 22$ with each asymmetric unit containing one $\alpha_{2} \beta \gamma$ heterotetramer. The four polypeptide chains of the heterotetramer are largely well defined with good electron density except for a few N-terminal and/or C-terminal residues, and the $\alpha, \beta$, and $\gamma$ subunits can be distinguished unambiguously based on the differences of numerous residues with large side chains (Fig. S3). There are no ligands bound at the active sites, the allosteric site, and the pseudo allosteric site; thus, this structure represents the structure of the IDH3 holoenzyme in apo form. It is noteworthy that the C-terminal region of the $\beta$ subunit is located on the structure surface and is involved in the crystal packing, which explains why the crystals of the $\beta$ mutant IDH3 holoenzyme diffracted 
134 X-rays better than the crystals of the wild-type IDH3 holoenzyme.

135

136

137

Table 1. Statistics of X-ray diffraction data and structure refinement.

\begin{tabular}{|c|c|}
\hline Structure & IDH3 Holoenzyme \\
\hline \multicolumn{2}{|l|}{ Diffraction data } \\
\hline Wavelength $(\AA)$ & 0.9792 \\
\hline Space group & $I 4_{1} 22$ \\
\hline \multicolumn{2}{|l|}{ Cell parameters } \\
\hline$a, b, c(\AA)$ & $204.57,204.57,237.88$ \\
\hline Resolution $(\AA)$ & $50.0-3.47(3.59-3.47)$ \\
\hline Observed reflections & 585,722 \\
\hline Unique reflections $(I / \sigma(I)>0)$ & 32,854 \\
\hline Average redundancy & $17.8(17.8)$ \\
\hline Average $\mathrm{I} / \sigma(\mathrm{I})$ & $35.1(1.7)$ \\
\hline Completeness (\%) & $100.0(100.0)$ \\
\hline $\mathrm{R}_{\text {merge }}(\%)$ & $10.1(187.5)$ \\
\hline CC $1 / 2(\%)$ & $99.5(67.6)$ \\
\hline \multicolumn{2}{|l|}{ Refinement and structure model } \\
\hline No. of reflections $(\mathrm{Fo}>0 \sigma(\mathrm{Fo}))$ & 30,525 \\
\hline Working set & 28,955 \\
\hline Test set & 1,570 \\
\hline $\mathrm{R}_{\text {work }} / \mathrm{R}_{\text {free }}$ factor & $0.21 / 0.25$ \\
\hline Total atoms & 9,851 \\
\hline Wilson B factor $\left(\AA^{2}\right)$ & 51.8 \\
\hline Average B factor $\left(\AA^{2}\right)$ & 56.1 \\
\hline \multicolumn{2}{|l|}{ RMS deviations } \\
\hline Bond lengths $(\AA)$ & 0.012 \\
\hline Bond angles $\left(^{\circ}\right)$ & 1.4 \\
\hline \multicolumn{2}{|l|}{ Ramachandran plot (\%) } \\
\hline Most favored & 85.8 \\
\hline Allowed & 14.2 \\
\hline Disallowed & 0.0 \\
\hline
\end{tabular}


Figure 1.
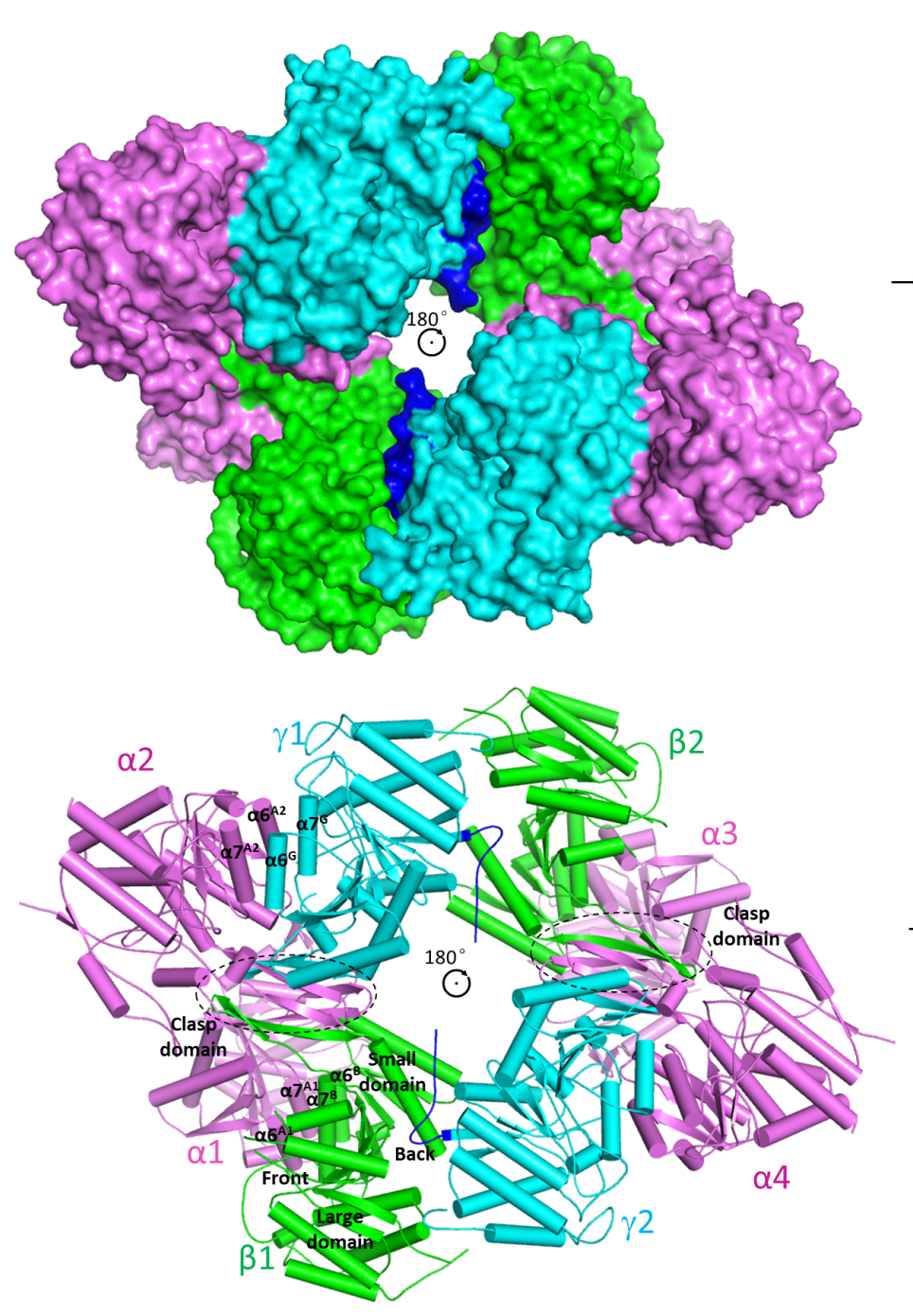

- $\gamma$ subunit $-\mathrm{N}$-terminal of $\gamma$ subunit

138
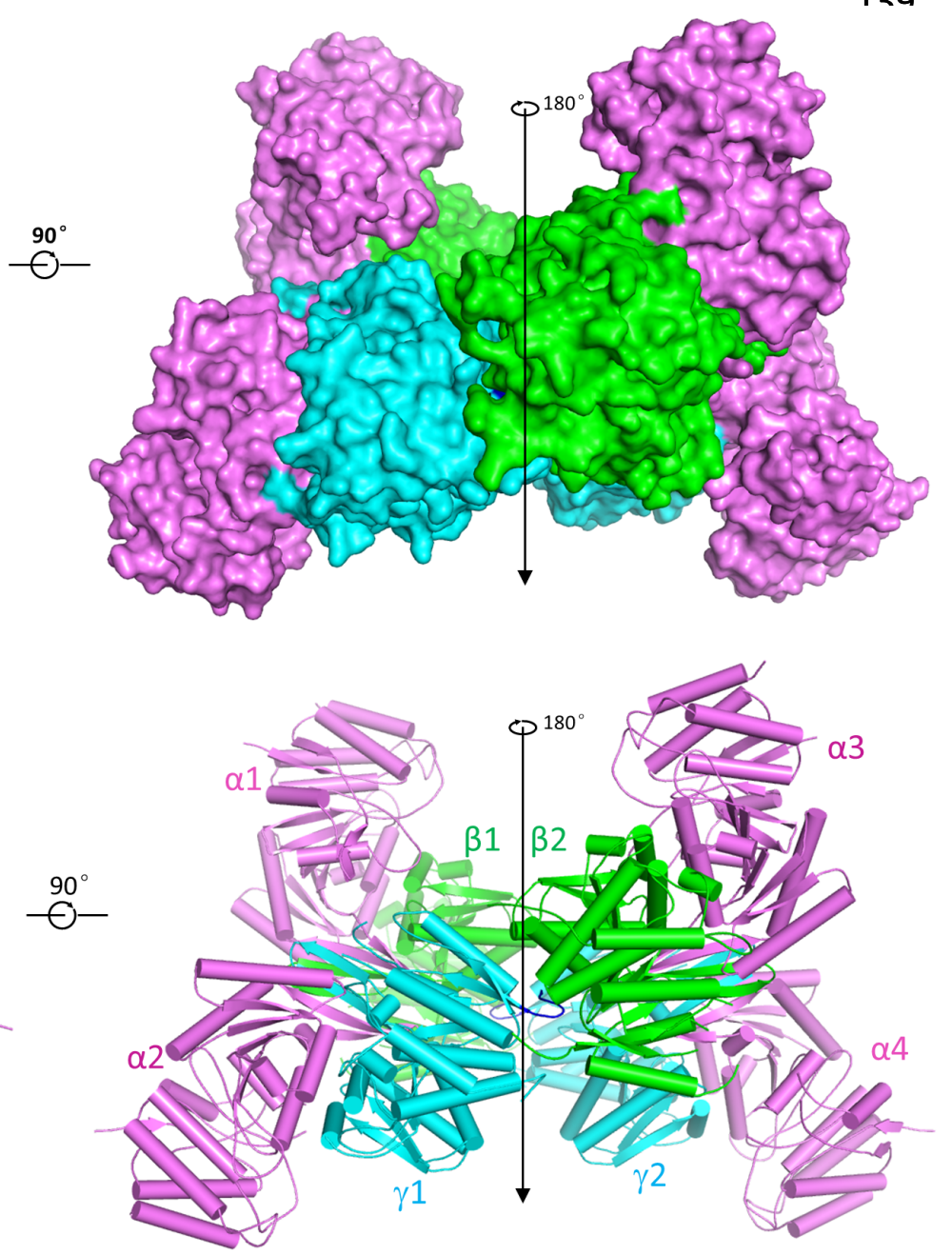

Figure 1. Overall structure of human IDH3 holoenzyme.

(A) Surface and (B) cartoon presentations of human IDH3 holoenzyme in two different orientations. Left: view along the crystallographic 2-fold axis of the IDH3 holoenzyme. Right: view in perpendicular to the crystallographic 2-fold axis of the IDH3 holoenzyme. The $\alpha, \beta$ and $\gamma$ subunits are colored in magenta, green and cyan, respectively. The large domain, small domain and clasp domain, and the front and back clefts of the $\beta$ subunit are indicated. The clasp domains of the $\alpha \beta$ or $\alpha \gamma$ heterodimers are indicated with dashed ovals, and the N-terminal regions of the $\gamma$ subunits are colored in blue. 
In the holoenzyme, the $\alpha \beta$ and $\alpha \gamma$ heterodimers assume very similar overall structures as those in the isolated forms (Ma et al., 2017a; Sun et al., 2019) (Fig. 1). Both heterodimers have a pseudo two-fold symmetry along the heterodimer interface. The heterodimer interface is mediated by the $\alpha 6$ and $\alpha 7$ helices of the small domains which form a four-helix bundle in a parallel manner, and the $\beta 6$ and $\beta 7$ strands of the clasp domains (the clasp $\beta$-strands) which form a four-stranded $\beta$-sheet (the clasp $\beta$-sheet) in an antiparallel manner. The heterodimer interface buries about $2180 \AA^{2}$ and $2094 \AA^{2}$ solvent accessible surface or $13.7 \%$ and $13.5 \%$ of the surface area of each subunit in the $\alpha \beta$ and $\alpha \gamma$ heterodimers, respectively, indicating that the heterodimer interface is very tight in both heterodimers.

Nevertheless, the $\alpha \beta$ and $\alpha \gamma$ heterodimers in the holoenzyme also exhibit some notable differences from those in the isolated forms. In particular, the $\alpha \beta$ heterodimer assumes an open overall conformation similar to that of the isolated $\alpha \gamma$ heterodimer rather than the compact conformation of the isolated $\alpha \beta$ heterodimer, rendering it suitable for allosteric activation and catalytic reaction (see discussion later). In addition, the $\mathrm{N}$-terminal region (residues 1-14) of the $\gamma$ subunit is disordered in all of our previously determined $\alpha \gamma$ structures regardless of the presence or absence of ligands; however, a large portion of the N-terminal region (residues 5-14) of the $\alpha \gamma$ heterodimer is well defined in this structure, which plays an important role in the formation and function of the heterooctamer (see discussion later).

\section{The heterodimer-heterodimer interface in the $\alpha_{2} \beta \gamma$ heterotetramer}

In the holoenzyme, the $\alpha_{2} \beta \gamma$ heterotetramer is assembled by the $\alpha \beta$ and $\alpha \gamma$ heterodimers via their clasp domains (Figs. 1 and 2A). There is a pseudo two-fold symmetry along the heterodimer-heterodimer interface, which is about $25^{\circ}$ off the coplane axis of the $\alpha \beta$ or $\alpha \gamma$ heterodimer. In other words, the coplane axes of the $\alpha \beta$ and $\alpha \gamma$ heterodimers make a $50^{\circ}$ angle. Thus, the heterotetramer has a distorted tetrahedron architecture with the two $\alpha$ subunits occupying two vertices on the same side and the $\beta$ and $\gamma$ subunits two vertices on the other side (Fig. 2A). The heterodimer-heterodimer interface buries about $804 \AA^{2}$ solvent accessible surface or $3.0 \%$ of the surface area of each heterodimer. At the interface, the clasp $\beta$-sheets of the two heterodimers interact with each other to form a $\beta$-barrel in a reciprocal manner such that the clasp $\beta$-strands of the $\beta$ subunit stack antiparallelly onto those of the $\alpha$ subunit of the $\alpha \gamma$ heterodimer, and the clasp $\beta$-strands of the $\gamma$ subunit stack antiparallelly onto those of the $\alpha$ subunit of the $\alpha \beta$ heterodimer (Fig. 2A). The interface consists of twenty-two hydrophobic residues and two Ser residues from the four clasp domains which form extensive hydrophobic interactions (Fig. 2B). In addition, there are eight hydrophilic residues which form two layers of hydrogen-bonding interactions to separate the hydrophobic 
interactions (Figs. 2B and 2C). Specifically, the side chains of His $131^{\mathrm{A} 1}$ and $\mathrm{Gln} 139^{\mathrm{A} 1}$ of the $\alpha$ subunit in the $\alpha \beta$ heterodimer and His $131^{\mathrm{A} 2}$ and $\mathrm{Gln} 139^{\mathrm{A} 2}$ of the $\alpha$ subunit in the $\alpha \gamma$

198 heterodimer form one network of hydrogen bonds, and the side chains of $G \ln 150^{\mathrm{B}}$ and 199 His $142^{\mathrm{B}}$ of the $\beta$ subunit and $\mathrm{G} \ln 148^{\mathrm{G}}$ and His $140^{\mathrm{G}}$ of the $\gamma$ subunit form another network of 200 hydrogen bonds (residues and structure elements of the $\alpha$ and $\beta$ subunits of the $\alpha \beta$ 201 heterodimer and the $\alpha$ and $\gamma$ subunits of the $\alpha \gamma$ heterodimer are superscripted by "A1" and 202 "B", and A2" and "G", respectively).

\section{The heterotetramer-heterotetramer interface in the $\left(\alpha_{2} \beta \gamma\right)_{2}$ heterooctamer}

205 In the holoenzyme, the $\left(\alpha_{2} \beta \gamma\right)_{2}$ heterooctamer is assembled by two $\alpha_{2} \beta \gamma$ heterotetramers related by a crystallographic two-fold symmetry via the $\beta$ and $\gamma$ subunits (Fig. 1). Thus, the heterooctamer has a distorted tetrahedron architecture, in which the two $\beta$ and two $\gamma$ subunits are arranged alternately to form the inner core, and the four $\alpha$ subunits are positioned on the periphery. The heterotetramer-heterotetramer interface buries about $2248 \AA^{2}$ solvent accessible surface or $4.3 \%$ of the surface area of the heterotetramer. At the interface, the $\mathrm{N}$-terminal region of the $\gamma$ subunit of one heterotetramer intrudes into a shallow cleft between the small and large domains of the $\beta$ subunit on the back of the pseudo allosteric site (designated as the "back cleft") of the other heterotetramer (Fig. 2D). In particular, residues 10-14 (AKYGG) of the $\gamma$ subunit make a number of hydrogen-bonding interactions with several residues of the back cleft of the $\beta$ subunit, which form a major part of the heterotetramer-heterotetramer interface (Fig. 2E). Specifically, the main-chain carbonyl of $\operatorname{Pro} 8^{\mathrm{G}}$ forms a hydrogen bond with the side chain of Lys $165^{\mathrm{B}}$; the side chain of Tyr $12^{\mathrm{G}}$ forms a hydrogen bond with the side chain of Asp $169^{\mathrm{B}}$ and additionally a $\pi-\pi$ stacking interaction with the side chain of Phe $166^{\mathrm{B}}$; the main-chain amine and carbonyl of Gly13 ${ }^{\mathrm{G}}$ form a hydrogen bond each with the main-chain carbonyl of Ala $264^{\mathrm{B}}$ and the side chain of His $301^{\mathrm{B}}$, respectively; the main-chain amine of $\mathrm{Gly} 14^{\mathrm{G}}$ forms a hydrogen bond with the main-chain carbonyl of Glu265 ${ }^{\mathrm{B}}$. In addition to the N-terminal region, the $\alpha 2$ helix of the $\gamma$ subunit also makes interactions with the $\alpha 4$ and $\alpha 5$ helices of the $\beta$ subunit, which form a minor part of the heterotetramer-heterotetramer interface. In this region, the main-chain carbonyl and side chain of $\operatorname{Arg} 70^{\mathrm{G}}$ on the $\alpha 2^{\mathrm{G}}$ helix form a hydrogen bond each with the side chain of Lys $165^{\mathrm{B}}$ and the main-chain carbonyl of Leu $205^{\mathrm{B}}$, respectively. Sequence alignments showed that although the $\mathrm{N}$-terminal region of the $\gamma$ subunit is different from that of the $\alpha$ and $\beta$ subunits, residues 10-14 (AKYGG) are strictly conserved in other mammalian NAD-IDHs and highly conserved in the regulatory subunit IDH1 of yeast NAD-IDH (Liu et al., 2018; Sun et al., 2019), suggesting that the N-terminal region of the regulatory subunit in other eukaryotic NAD-IDHs might play a similar role in the assembly of the holoenzyme. 
Figure 2.

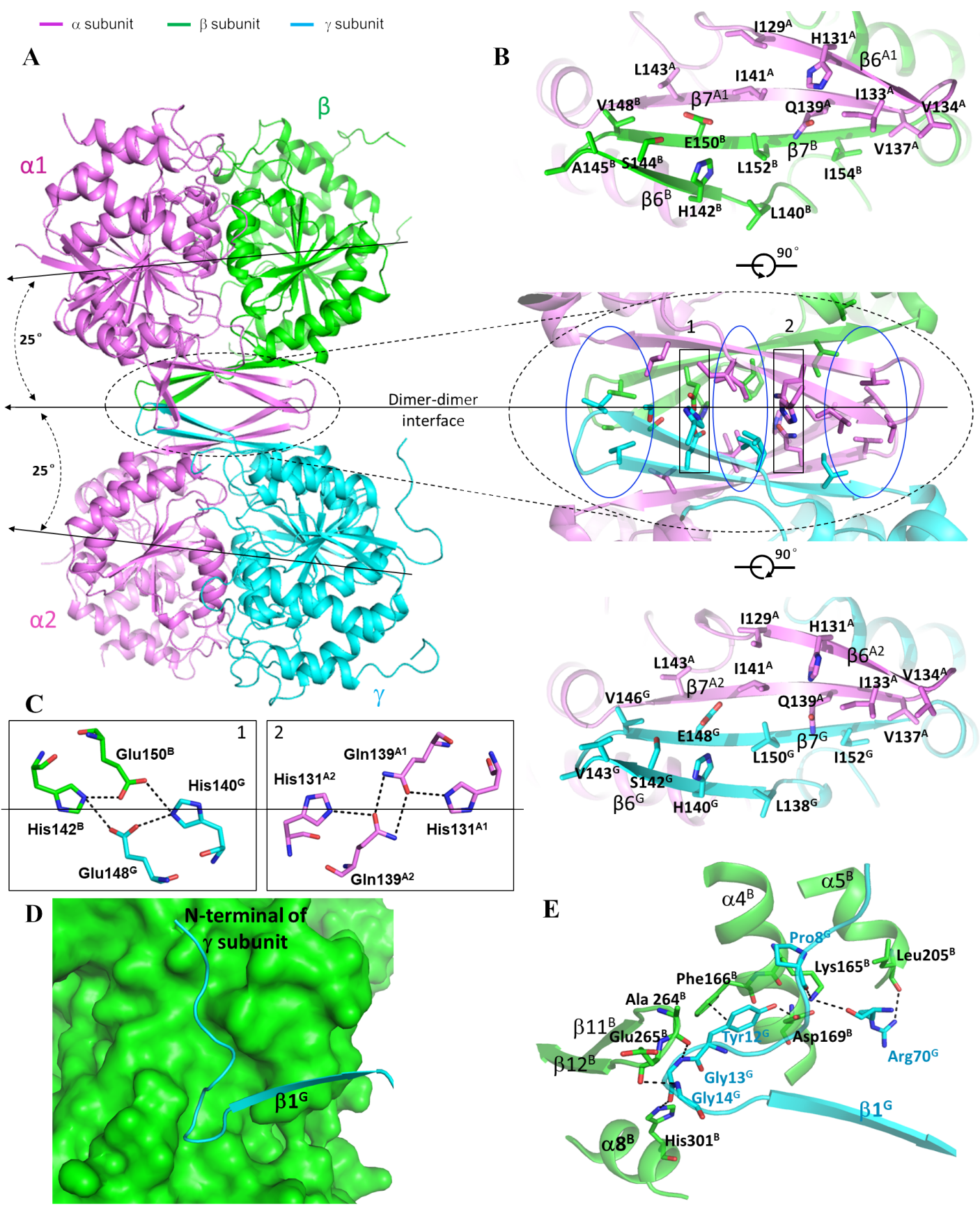

Figure 2. Interactions at the heterodimer-heterodimer and the heterotetramer-heterotetramer 234 interfaces.

235 (A) The $\alpha_{2} \beta \gamma$ heterotetramer is assembled by the $\alpha \beta$ and $\alpha \gamma$ heterodimers via their clasp domains. The 236 color coding of the $\alpha, \beta$ and $\gamma$ subunits is the same as in Figure 1. The pseudo 2-fold axis along the 237 heterodimer-heterodimer interface, and the coplane axes of the $\alpha \beta$ and $\alpha \gamma$ heterodimers are indicated.

238 (B) Structure of the heterodimer-heterodimer interface. Middle panel: interactions at the interface 239 consist of largely hydrophobic residues (marked by blue ovals) and a few hydrophilic residues (marked 240 by black rectangles). Upper panel: interactions between the $\alpha$ and $\beta$ subunits at the interface. Lower 241 panel: interactions between the $\alpha$ and $\gamma$ subunits at the interface. (C) Hydrogen-bonding interactions 
243 diagram showing that the $\mathrm{N}$-terminal region of the $\gamma$ subunit (in blue ribbon) of one heterotetramer

244 intrudes into the back cleft of the $\beta$ subunit (in green surface) of the other heterotetramer. (E)

245 Interactions between the N-terminal of the $\gamma$ subunit and the back cleft of the $\beta$ subunit. The

246 hydrogen-bonding interactions are indicated with dashed lines.

\section{The apo IDH3 holoenzyme assumes an inactive conformation}

249 Structural comparison shows that the $\alpha \gamma$ heterodimer in the apo IDH3 holoenzyme adopts an

250 inactive overall conformation similar to that in the isolated $\alpha^{\mathrm{Mg}} \gamma$ heterodimer. Specifically, the

251 key residues at the active site $\left(\operatorname{Tyr} 126^{\mathrm{A} 2}\right)$ and the allosteric site $\left(\operatorname{Tyr} 135^{\mathrm{G}}\right)$ assume similar

252 conformations as those in the inactive $\alpha^{\mathrm{Mg}} \gamma$ structure rather than those in the active

$253 \alpha^{\mathrm{Mg}} \gamma^{\mathrm{Mg}+\mathrm{CIT}+\mathrm{ADP}}$ structure; the N-terminal regions of both $\alpha 7^{\mathrm{A} 2}$ and $\alpha 7^{\mathrm{G}}$ helices at the

254 heterodimer interface assume the inactive loop conformations; and additionally, the allosteric

255 site is in proper conformation to bind the activators (Fig. 3A). Intriguingly, the $\alpha \beta$

256 heterodimer in the apo IDH3 holoenzyme exhibits some conformational differences compared

257 with the isolated $\alpha^{\mathrm{Ca}} \beta$ heterodimer. Structural analysis reveals that the insertion of the

258 N-terminus of the $\gamma$ subunit into the back cleft of the $\beta$ subunit pushes the large domain of the

$259 \beta$ subunit to rotate away from the $\alpha$ subunit (the structure elements moving away from the $\alpha$

260 subunit by about 1.5-3 $\AA$ ) (Fig. 3B). Consequently, the $\alpha \beta$ heterodimer assumes an open

261 overall conformation similar to that of the $\alpha^{\mathrm{Mg}} \gamma$ structure rather than the compact

262 conformation of the isolated $\alpha^{\mathrm{Ca}} \beta$ structure. In particular, the key residues at the active site

$263\left(\operatorname{Tyr} 126^{\mathrm{Al}}\right)$ and the pseudo allosteric site $\left(\operatorname{Tyr} 137^{\mathrm{B}}\right)$ assume inactive conformations similar to

264 those in the $\alpha^{\mathrm{Mg}} \gamma$ structure (Fig. 3C). In addition, the N-terminal region of helix $\alpha 7^{\mathrm{A} 1}$ of the $\alpha$

265 subunit at the heterodimer interface assumes a loop conformation; however, the N-terminal

266 region of helix $\alpha 7^{\mathrm{B}}$ of the $\beta$ subunit assumes a helix conformation with unknown reason.

267 At the pseudo allosteric site, the $\beta 3^{\mathrm{B}}-\alpha 3^{\mathrm{B}}$ loop is disordered, similar to that in the isolated

$268 \alpha^{\mathrm{Ca}} \beta$ structure. On the other hand, the $\beta 12^{\mathrm{B}}-\alpha 8^{\mathrm{B}}$ loop exhibits some conformational difference

269 from that in the isolated $\alpha^{\mathrm{Ca}} \beta$ structure (Fig. 3D). Nevertheless, the $\mathrm{N}$-terminal region of the

$270 \beta 12^{\mathrm{B}}-\alpha 8^{\mathrm{B}}$ loop maintains interactions with the $\alpha 6^{\mathrm{A} 1}$ and $\alpha 7^{\mathrm{B}}$ helices at the heterodimer

271 interface and still occupies the ADP-binding site in the $\alpha^{\mathrm{Mg}} \gamma^{\mathrm{Mg}+\mathrm{CIT}+\mathrm{ADP}}$ structure, prohibiting

272 the ADP binding. These results together indicate that the formation of the heterooctamer

273 renders the $\alpha \beta$ heterodimer to adopt an overall conformation similar to that of the $\alpha \gamma$

274 heterodimer; however, the pseudo allosteric site remains incapable of binding the activators

275 and thus the $\beta$ subunit still has no regulatory function. This explains why the $\alpha \beta$ heterodimer

276 in the holoenzyme can be allosterically activated and has normal enzymatic activity but

277 cannot bind the activators. These results also demonstrate that the structure characteristics and 
the regulatory mechanisms of the $\alpha \beta$ and $\alpha \gamma$ heterodimers uncovered from the structure and

279 biochemical studies of the isolated heterodimers are largely applicable to the holoenzyme.

Figure 3.

A

$$
-\alpha \text { subunit }-\beta \text { subunit }-\gamma \text { subunit }
$$
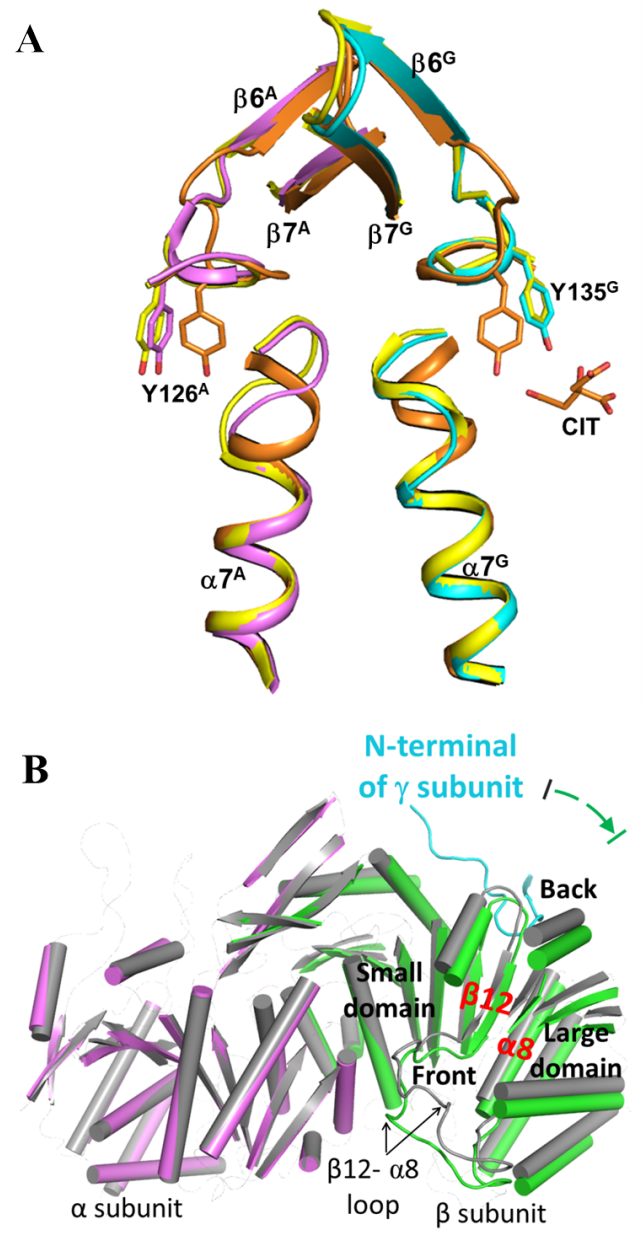

D

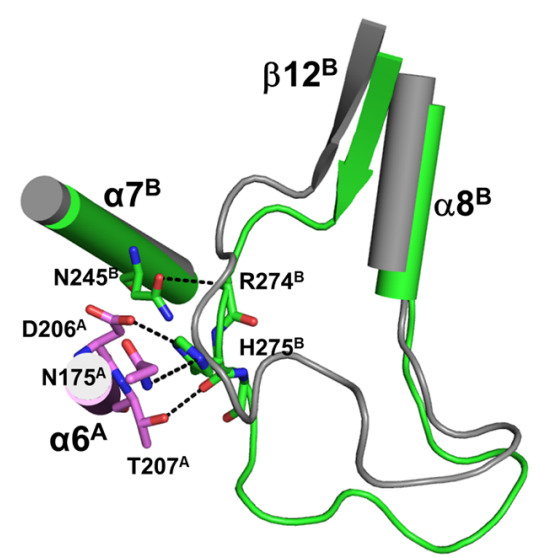

C $-\alpha^{M g} \gamma-\alpha^{M g} \gamma^{M g+C I T+A D P}=\alpha^{C a \beta}$
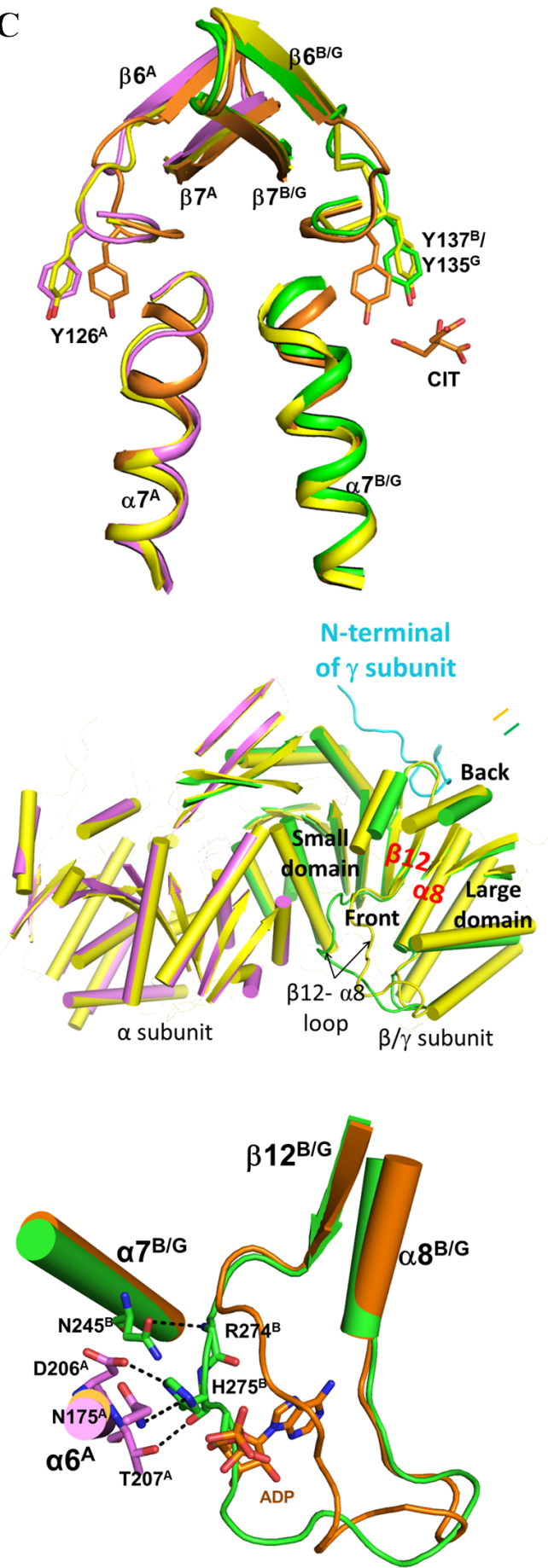

Figure 3. Structural comparisons of the $\alpha \beta$ and $\alpha \gamma$ heterodimers in the apo holoenzyme and in the isolated forms.

283 (A) Comparison of the $\alpha \gamma$ heterodimer in the holoenzyme and in the isolated forms. The color coding 284 of the subunits and structures is shown above. The key residues at the active site (Tyr $\left.126^{A}\right)$ and the 
allosteric site $\left(\operatorname{Tyr} 135^{\mathrm{G}}\right)$ assume similar conformations as those in the inactive $\alpha^{\mathrm{Mg}} \gamma$ structure rather than those in the active $\alpha^{\mathrm{Mg}} \gamma^{\mathrm{Mg}+\mathrm{CIT}+\mathrm{ADP}}$ structure. (B) Comparison of the overall conformation of the $\alpha \beta$ heterodimer in the holoenzyme with that of the isolated $\alpha^{\mathrm{Ca}} \beta$ heterodimer (colored in gray, left panel) and $\alpha^{\mathrm{Mg}} \gamma$ heterodimer (colored in yellow, right panel). The $\alpha \beta$ heterodimer assumes an open overall conformation similar to that of the isolated $\alpha^{\mathrm{Mg}} \gamma$ heterodimer rather than the compact conformation of the isolated $\alpha^{\mathrm{Ca}} \beta$ heterodimer. For clarity, only the $\alpha$ helices and $\beta$ strands are shown, and the loops are omitted except the $\beta 12-\beta 8$ loops of the $\beta$ and $\gamma$ subunits. The $\mathrm{N}$-terminal of the $\gamma$ subunit from another heterotetramer is also shown. (C) Comparison of the $\alpha \beta$ heterodimer in the holoenzyme with the isolated $\alpha^{\mathrm{Mg}} \gamma$ heterodimer and $\alpha^{\mathrm{Mg}} \gamma^{\mathrm{Mg}+\mathrm{CIT}+\mathrm{ADP}}$ heterodimer. The key residues at the active site (Tyr126 and the allosteric site $\left(\operatorname{Tyr} 137^{\mathrm{B}}\right)$ assume similar conformations as those in the inactive $\alpha^{\mathrm{Mg}} \gamma$ structure rather than those in the active $\alpha^{\mathrm{Mg}} \gamma^{\mathrm{Mg}+\mathrm{CIT}+\mathrm{ADP}}$ structure. (D) The $\beta 12-\beta 8$ loop of the $\beta$ subunit in the holoenzyme exhibits some conformational differences from that in the isolated $\alpha^{\mathrm{Ca}} \beta$ heterodimer but still occupies the ADP-binding site in the $\alpha^{\mathrm{Mg}} \gamma^{\mathrm{Mg}+\mathrm{CIT}+\mathrm{ADP}}$ structure. The hydrogen-bonding interactions of the $\beta 12-\beta 8$ loop with the $\alpha 7^{\mathrm{B}}$ and $\alpha 6^{\mathrm{A}}$ helices are indicated with dashed lines.

The clasp domains of the $\alpha \beta$ and $\alpha \gamma$ heterodimers play a critical role in the assembly and allosteric regulation of the $\alpha_{2} \beta \gamma$ heterotetramer

Our previous biochemical studies showed that the holoenzyme has notably higher activity than the sum of the isolated $\alpha \beta$ and $\alpha \gamma$ heterodimers in both absence and presence of the activators, suggesting that in the holoenzyme, both $\alpha \beta$ and $\alpha \gamma$ heterodimers are allosterically activated and exert catalytic function (Ma et al., 2017b). Our biochemical and structural studies of the $\alpha \gamma$ heterodimer showed that residues $\operatorname{Arg} 97^{\mathrm{G}}, \operatorname{Tyr} 135^{\mathrm{G}}$, and $\operatorname{Arg} 272^{\mathrm{G}}$ at the allosteric site, and residues $\operatorname{Lys} 151^{\mathrm{G}}$ and $\mathrm{Lys} 142^{\mathrm{A} 2}$ at the heterodimer interface play important roles in the allosteric activation (Ma et al., 2017a). Structural analysis of the apo IDH3 holoenzyme shows that residues His $131^{\mathrm{A} 1}, \mathrm{Gln} 139^{\mathrm{A} 1}$, His $131^{\mathrm{A} 2}, \mathrm{Gln} 139^{\mathrm{A} 2}, \mathrm{His}_{142^{\mathrm{B}}}, \mathrm{Glu} 150^{\mathrm{B}}$, His $140^{\mathrm{G}}$, and Glu $148^{\mathrm{G}}$ of the clasp domains play an important role in the assembly of the $\alpha_{2} \beta \gamma$ heterotetramer. To investigate the functional roles of these residues in the holoenzyme, we prepared a series of mutant holoenzymes containing mutations of the key residues at the allosteric site $\left(\gamma_{\mathrm{R} 97 \mathrm{~A}}, \gamma_{\mathrm{Y} 135 \mathrm{~A}}\right.$, and $\left.\gamma_{\mathrm{R} 272 \mathrm{~A}}\right)$, the pseudo allosteric site $\left(\beta_{\mathrm{R} 99 \mathrm{~A}}, \beta_{\mathrm{Y} 137 \mathrm{~A}}\right.$, and $\beta_{\mathrm{R} 274 \mathrm{~A}}$, corresponding to $\gamma_{\mathrm{R} 97 \mathrm{~A}}, \gamma_{\mathrm{Y} 135 \mathrm{~A}}$, and $\left.\gamma_{\mathrm{R} 272 \mathrm{~A}}\right)$, the heterodimer interfaces $\left(\alpha 1_{\mathrm{K} 142 \mathrm{~A}}, \alpha 2_{\mathrm{K} 142 \mathrm{~A}}\right.$, $\alpha 1_{\mathrm{K} 142 \mathrm{~A}} \alpha 2_{\mathrm{K} 142 \mathrm{~A}}, \quad \beta_{\mathrm{K} 153 \mathrm{~A}}$, and $\left.\gamma_{\mathrm{K} 151 \mathrm{~A}}\right)$, and the heterodimer-heterodimer interface $\left(\alpha 1_{\mathrm{Q} 139 \mathrm{~A}}\right.$, $\alpha 2_{\mathrm{Q} 139 \mathrm{~A}}, \alpha 1_{\mathrm{Q} 139 \mathrm{~A}} \alpha 2_{\mathrm{Q} 139 \mathrm{~A}}, \beta_{\mathrm{E} 150 \mathrm{~A}}$, and $\left.\gamma_{\mathrm{E} 148 \mathrm{~A}}\right)$, and measured their kinetic parameters in the 317 absence or presence of CIT and ADP to examine the effects of the mutations on the activity 318 and allosteric activation of the holoenzyme. The mutant $\alpha \beta$ and $\alpha \gamma$ heterodimers containing mutations $\alpha 1_{\mathrm{H} 131 \mathrm{~A}}, \alpha 2_{\mathrm{H} 131 \mathrm{~A}}, \beta_{\mathrm{H} 142 \mathrm{~A}}$, and $\gamma_{\mathrm{H} 140 \mathrm{~A}}$ could not be expressed for unknown reason(s) and thus the mutant holoenzymes containing these mutations could not be obtained. 
The wild-type holoenzyme exhibits a $V_{\max }$ of $28.6 \mu \mathrm{mol} / \mathrm{mg} / \mathrm{min}$ and a $S_{0.5, \mathrm{ICT}}$ of 3.54 $\mathrm{mM}$ in the absence of the activators and a $V_{\max }$ of $30.6 \mu \mathrm{mol} / \mathrm{mg} / \mathrm{min}$ and a $S_{0.5, \mathrm{ICT}}$ of $0.43 \mathrm{mM}$ in the presence of the activators, and displays a significant activation effect ( 8.2 folds) 324 (defined as the ratio of the $S_{0.5, \text { ICT }}$ in the presence and absence of the activators) (Table 2 and 325 Fig. 4). Compared to the wild-type holoenzyme, the mutant holoenzymes containing 326 mutations of the key residues at the allosteric site $\left(\gamma_{\mathrm{R} 97 \mathrm{~A}}, \gamma_{\mathrm{Y} 135 \mathrm{~A}}\right.$, and $\left.\gamma_{\mathrm{R} 272 \mathrm{~A}}\right)$ exhibit comparable $327 V_{\max }\left(<1.2\right.$ folds) and $S_{0.5, \text { ICT }}(<1.6$ folds $)$ in the absence of the activators, and display weak or 328 no activation effects (0.9-2.7 folds), indicating that the mutations at the allosteric site have 329 significant impacts on the activation of the holoenzyme (Table 2 and Fig. 4). On the other 330 hand, the mutant holoenzymes containing mutations of the key residues at the pseudo 331 allosteric site $\left(\beta_{\mathrm{R} 99 \mathrm{~A}}, \beta_{\mathrm{Y} 137 \mathrm{~A}}\right.$, and $\left.\beta_{\mathrm{R} 274 \mathrm{~A}}\right)$ also exhibit comparable $V_{\max }(<1.3$ folds $)$ but slightly 332 decreased $S_{0.5 \text {, ICT }}(<3.9$ folds) in the absence of the activators, and display moderate activation 333 effects (3.1-4.3 folds), indicating that the mutations at the pseudo allosteric site have insignificant impacts on the activation and function of the holoenzyme (Table 2 and Fig. 4).

The mutant holoenzymes containing mutations of the key residues at the heterodimer interfaces $\left(\alpha 1_{\mathrm{K} 142 \mathrm{~A}}, \quad \alpha 2_{\mathrm{K} 142 \mathrm{~A}}, \quad \alpha 1_{\mathrm{K} 142 \mathrm{~A}} \alpha 2_{\mathrm{K} 142 \mathrm{~A}}, \quad \beta_{\mathrm{K} 153 \mathrm{~A}}, \quad\right.$ and $\left.\gamma_{\mathrm{K} 151 \mathrm{~A}}\right)$ exhibit significantly decreased $V_{\max }$ (3.1-15.1 folds) and a varied $S_{0.5}$ (0.4-3.2 folds) in the absence of the activators, and display moderate or no activation effects (0.7-3.3 folds) (Table 2 and Fig. 4). In particular, the mutant holoenzyme containing the $\gamma_{\mathrm{K} 151 \mathrm{~A}}$ mutation completely disrupts the activation. These results indicate that the mutations at the heterodimer interface significantly impair the communication from the allosteric site to the active sites of both $\alpha$ subunits and thus have severe impacts on the activation and function of the holoenzyme. For the key residues at the heterodimer-heterodimer interface, the mutant holoenzymes containing 344 mutations $\beta_{\mathrm{E} 150 \mathrm{~A}}$ and $\gamma_{\mathrm{E} 148 \mathrm{~A}}$ exhibit slightly decreased $V_{\max }$ (about 2 folds) and $\mathrm{S}_{0.5}$ (about 2.7 345 folds) in the absence of the activators, and display substantial activation effects (5.2-5.6 folds), 346 indicating that these mutations have minor impacts on the activation and function of the 347 holoenzyme (Table 2 and Fig. 4). Intriguingly, the mutant holoenzymes containing mutations $348 \alpha 1_{\mathrm{Q} 139 \mathrm{~A}}, \alpha 2_{\mathrm{Q} 139 \mathrm{~A}}$, and $\alpha 1_{\mathrm{Q} 139 \mathrm{~A}} \alpha 2_{\mathrm{Q} 139 \mathrm{~A}}$ display slightly higher $V_{\max }$ (about 1.2-1.6 folds) but 349 significantly decreased $S_{0.5 \text {, Іст }}(6.8-14.8$ folds) in the absence of the activators, and display 350 weak activation effects $(<1.7$ folds $)$, indicating that these mutants are constitutively active regardless the absence or presence of the activators (Table 2 and Fig. 4).

Taken together, the biochemical data demonstrate that the allosteric site plays a critical role and the pseudo allosteric site has no regulatory role in the allosteric activation of the 354 holoenzyme; the heterodimer interfaces play a vital role in the allosteric regulation and 355 function of the holoenzyme; and the heterodimer-heterodimer interface plays an important 356 role in the assembly and allosteric regulation of the $\alpha_{2} \beta \gamma$ heterotetramer and the holoenzyme. 
358 Table 2. Activities and kinetic parameters of the wild-type and mutant IDH3 holoenzymes.

\begin{tabular}{|l|l|l|l|}
\hline Enzyme & $\begin{array}{l}V_{\text {max }}(\mathbf{m m o l} / \mathbf{m g} / \mathbf{m i n}) \\
\text {-activators/+activators }\end{array}$ & $\begin{array}{l}\boldsymbol{S}_{\mathbf{0 . 5}, \text { ICT }}(\mathbf{m M}) \\
\text {-activators/+activators }\end{array}$ & $\begin{array}{l}\text { Activation } \\
\text { effect }^{\text {a }} \text { (fold) }\end{array}$ \\
\hline$\alpha \beta$ & $2.72 \pm 0.14 / 2.80 \pm 0.23$ & $3.65 \pm 0.39 / 3.63 \pm 0.62$ & 1.0 \\
\hline$\alpha \gamma$ & $9.62 \pm 0.23 / 16.1 \pm 1.1$ & $5.42 \pm 0.71 / 0.26 \pm 0.07$ & 20.8 \\
\hline$\alpha \beta \alpha \gamma$ & $28.6 \pm 0.3 / 30.6 \pm 1.0$ & $3.54 \pm 0.18 / 0.43 \pm 0.03$ & 8.2 \\
\hline
\end{tabular}

Pseudo allosteric site

\begin{tabular}{|l|l|l|l|}
\hline$\alpha \beta_{\mathrm{R} 99 \mathrm{~A}} \alpha \gamma$ & $24.9 \pm 0.8 / 27.7 \pm 1.5$ & $1.45 \pm 0.07 / 0.34 \pm 0.05$ & 4.3 \\
\hline$\alpha \beta_{\mathrm{Y} 137 \mathrm{~A}} \alpha \gamma$ & $30.2 \pm 1.0 / 39.4 \pm 3.8$ & $1.50 \pm 0.09 / 0.49 \pm 0.05$ & 3.1 \\
\hline$\alpha \beta_{\mathrm{R} 274 \mathrm{~A}} \alpha \gamma$ & $35.9 \pm 1.2 / 38.0 \pm 2.4$ & $0.91 \pm 0.01 / 0.23 \pm 0.06$ & 4.0 \\
\hline
\end{tabular}

Allosteric site

\begin{tabular}{|l|l|l|l|}
\hline$\alpha \beta \alpha \gamma_{\mathrm{R} 97 \mathrm{~A}}$ & $33.2 \pm 0.8 / 34.4 \pm 2.1$ & $3.46 \pm 0.29 / 1.20 \pm 0.09$ & 2.9 \\
\hline$\alpha \beta \alpha \gamma_{\mathrm{Y} 135 \mathrm{~A}}$ & $27.8 \pm 1.1 / 33.2 \pm 4.2$ & $2.28 \pm 0.23 / 2.16 \pm 0.12$ & 1.1 \\
\hline$\alpha \beta \alpha \gamma_{\mathrm{R} 272 \mathrm{~A}}$ & $27.5 \pm 0.8 / 34.1 \pm 3.3$ & $2.40 \pm 0.20 / 2.80 \pm 0.30$ & 0.9 \\
\hline
\end{tabular}

Heterodimer interface

\begin{tabular}{|l|l|l|l|}
\hline$\alpha \beta_{\mathrm{K} 153 \mathrm{~A}} \alpha \gamma$ & $9.21 \pm 0.43 / 18.4 \pm 2.0$ & $3.13 \pm 0.49 / 1.80 \pm 0.13$ & 1.7 \\
\hline$\alpha \beta \alpha \gamma_{\mathrm{K} 151 \mathrm{~A}}$ & $5.24 \pm 0.63 / 9.19 \pm 2.12$ & $6.96 \pm 0.50 / 10.2 \pm 3.1$ & 0.7 \\
\hline$\alpha_{\mathrm{K} 142 \mathrm{~A}} \beta \alpha \gamma$ & $6.53 \pm 0.44 / 6.84 \pm 0.45$ & $2.50 \pm 0.35 / 0.76 \pm 0.03$ & 3.3 \\
\hline$\alpha \beta \alpha_{\mathrm{K} 142 \mathrm{~A}} \gamma$ & $3.33 \pm 0.11 / 4.20 \pm 0.61$ & $1.55 \pm 0.10 / 0.84 \pm 0.04$ & 1.8 \\
\hline$\alpha_{\mathrm{K} 142 \mathrm{~A}} \beta \alpha_{\mathrm{K} 142 \mathrm{~A}} \gamma$ & $1.92 \pm 0.40 / 1.48 \pm 0.22$ & $11.42 \pm 2.01 / 8.50 \pm 2.50$ & 1.3 \\
\hline
\end{tabular}

Heterodimer-heterodimer interface

\begin{tabular}{|l|l|l|l|}
\hline$\alpha \beta_{\mathrm{E} 150 \mathrm{~A}} \alpha \gamma$ & $15.2 \pm 0.7 / 25.1 \pm 1.0$ & $1.34 \pm 0.05 / 0.24 \pm 0.07$ & 5.6 \\
\hline$\alpha \beta \alpha \gamma_{\mathrm{E} 148 \mathrm{~A}}$ & $13.4 \pm 0.4 / 14.0 \pm 1.7$ & $1.29 \pm 0.05 / 0.25 \pm 0.12$ & 5.2 \\
\hline$\alpha_{\mathrm{Q} 139 \mathrm{~A}} \beta \alpha \gamma$ & $36.1 \pm 1.9 / 43.4 \pm 2.3$ & $0.52 \pm 0.04 / 0.35 \pm 0.04$ & 1.5 \\
\hline$\alpha \beta \alpha_{\mathrm{Q} 139 \mathrm{~A}} \gamma$ & $33.0 \pm 1.2 / 36.4 \pm 0.6$ & $0.40 \pm 0.05 / 0.23 \pm 0.03$ & 1.7 \\
\hline$\alpha_{\mathrm{Q} 139 \mathrm{~A}} \beta \alpha_{\mathrm{Q} 139 \mathrm{~A}} \gamma$ & $39.7 \pm 0.9 / 47.4 \pm 1.6$ & $0.24 \pm 0.03 / 0.18 \pm 0.03$ & 1.3 \\
\hline
\end{tabular}

Deletion of the N-terminal of the $\gamma$ subunit $(\Delta N)$

\begin{tabular}{|l|l|l|l|}
\hline$\alpha \gamma_{\Delta \mathrm{N}}$ & $9.51 \pm 0.16 / 18.2 \pm 0.47$ & $5.73 \pm 0.40 / 0.46 \pm 0.04$ & 12.5 \\
\hline$\alpha \beta \alpha \gamma_{\Delta \mathrm{N}}$ & $5.50 \pm 0.21 / 13.0 \pm 0.56$ & $3.85 \pm 0.43 / 2.02 \pm 0.09$ & 1.9 \\
\hline
\end{tabular}

359

\footnotetext{
${ }^{\text {a }}$ Activation effect $=S_{0.5, \text { ICT }}$ (no activators) $/ S_{0.5, \text { ICT }}$ (+activators).
}

The enzymatic activity and kinetic data were measured at standard conditions with varied concentrations of ICT in the absence or presence of the activators (CIT and ADP). 
Figure 4.

A

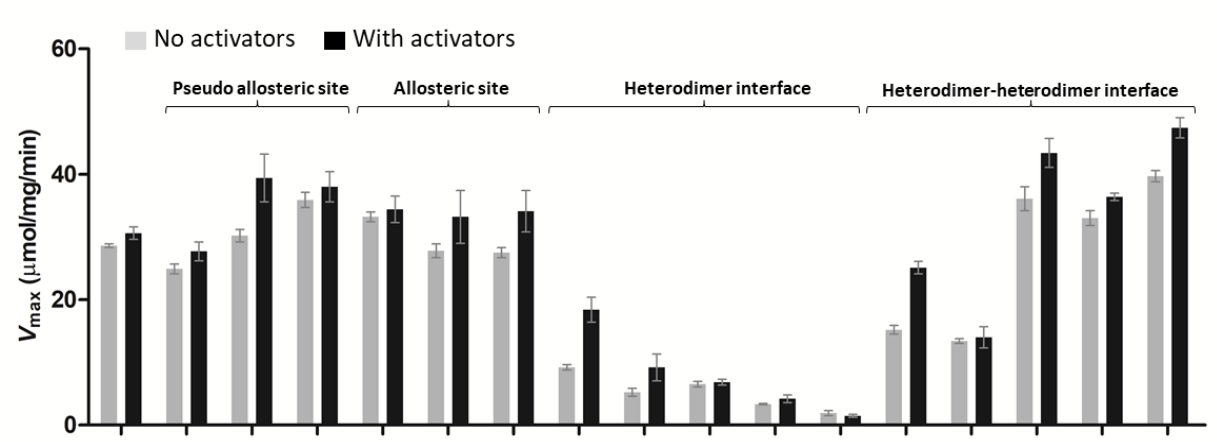

B
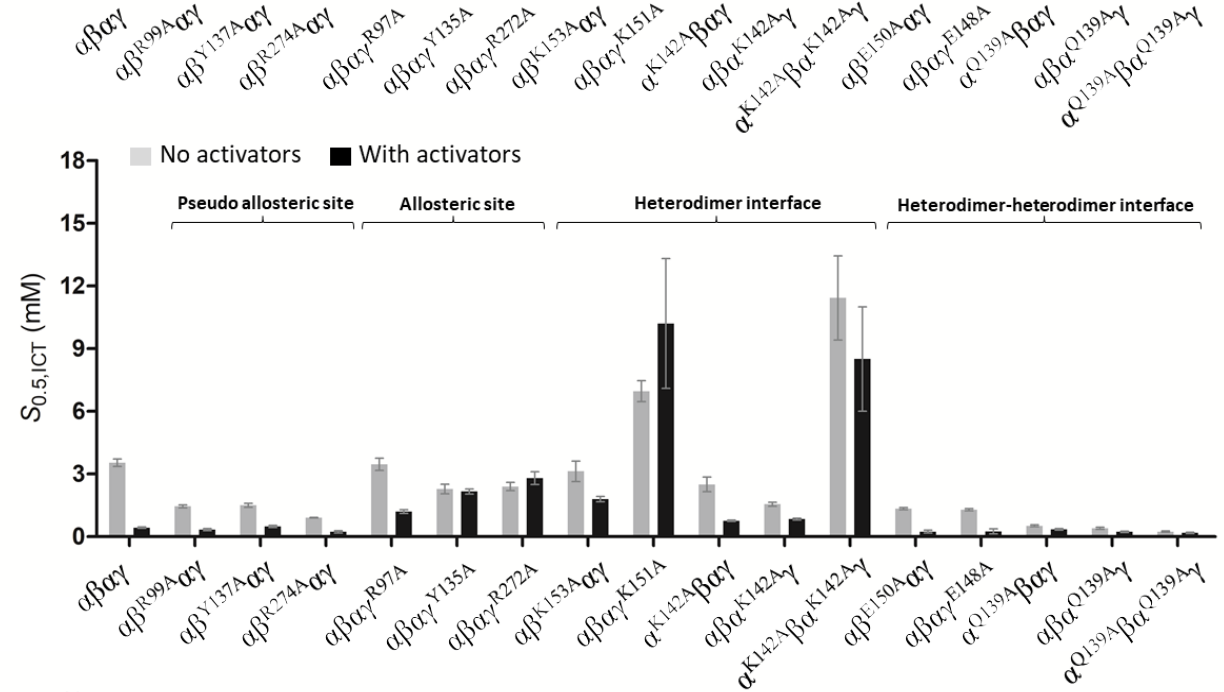

C

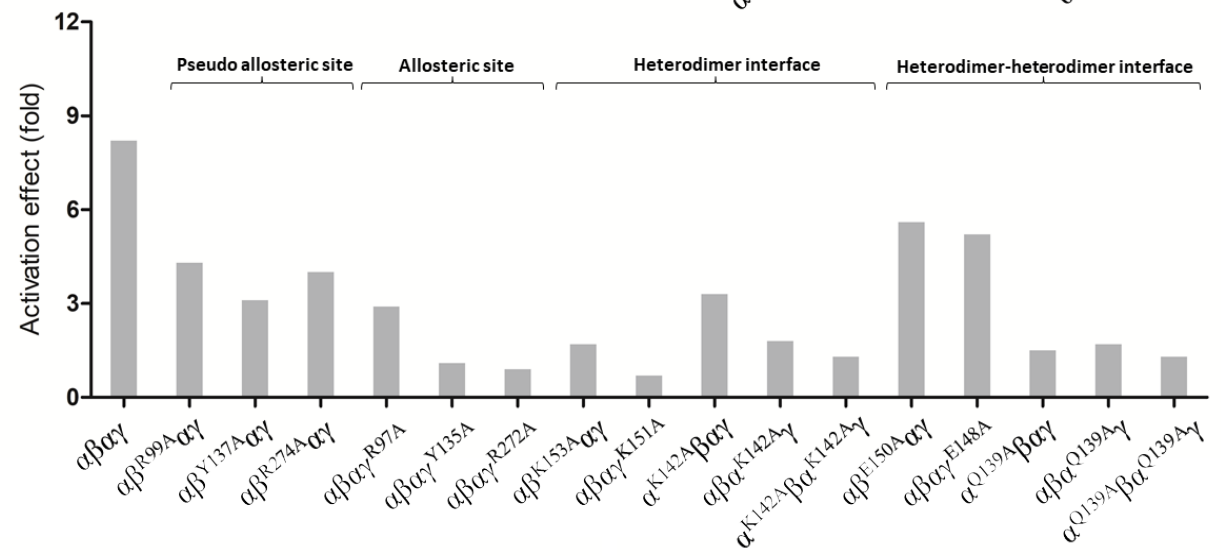

D
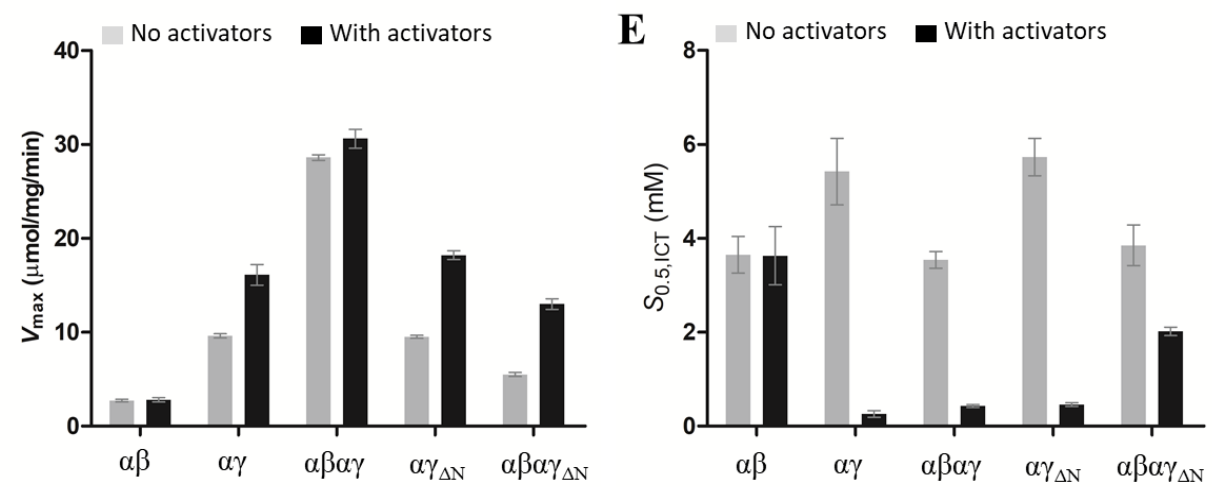

Figure 4. Effects of the mutations on the activity and allosteric activation of the IDH3 
367 (A) Graph presentations of the $V_{\max }$ values, (B) the $S_{0.5, \mathrm{ICT}}$ values, and (C) the activation effects of the 368 wild-type holoenzyme and mutant holoenzymes containing mutations of key residues at the allosteric 369 site, the pseudo allosteric site, the heterodimer interfaces, and the heterodimer-heterodimer interface in

370 the absence or presence of CIT and ADP. The activation effect is defined as the ratio of the $S_{0.5, \text { ICT }}$ in the absence and presence of the activators. The detailed kinetic parameters are listed in Table 2. (D) Graph presentations of the $V_{\max }$ values and (E) the $S_{0.5, \text { ICT }}$ values of the wild-type $\alpha \beta$ and $\alpha \gamma$

373 heterodimers and holoenzyme, and the mutant $\alpha \gamma$ heterodimer and holoenzyme with the N-terminal of 374 the $\gamma$ subunit removed $(\Delta \mathrm{N})$ in the absence and presence of CIT and ADP. The detailed kinetic 375 parameters are listed in Table 2.

The N-terminus of the $\gamma$ subunit is essential for the assembly and function of the holoenzyme

In the IDH3 holoenzyme, the N-terminal region of the $\gamma$ subunit of one heterotetramer inserts into the back cleft of the $\beta$ subunit of the other heterotetramer to form the heterooctamer. To validate the functional role of the $\mathrm{N}$-terminus of the $\gamma$ subunit in the assembly and function of the holoenzyme, we removed the N-terminal region (residues 1-14) of the $\gamma$ subunit $\left(\gamma_{\Delta \mathrm{N}}\right)$, and prepared the mutant $\alpha \gamma_{\Delta \mathrm{N}}$ heterodimer and $\alpha_{2} \beta \gamma_{\Delta \mathrm{N}}$ heterotetramer. The SEC-MALS analyses show that like the wild-type $\alpha \gamma$ heterodimer, the mutant $\alpha \gamma_{\Delta \mathrm{N}}$ heterodimer exists as a dimer with an average molecular weight of $84 \mathrm{kDa}$ at a low concentration $(2 \mathrm{mg} / \mathrm{ml})$ and a tetramer (presumably a dimer of heterodimers) with an average molecular weight of $123 \mathrm{kDa}$ at a high concentration (12 mg/ml) (Fig. S4A and Table S3). However, unlike the wild-type holoenzyme which exists as a stable octamer at both the low and high concentrations with an average molecular weight of about $284 \mathrm{kDa}$, the mutant $\alpha_{2} \beta \gamma_{\Delta \mathrm{N}}$ heterotetramer exhibits an average molecular weight of $106 \mathrm{kDa}$ and thus appears to be a mixture of the $\alpha \beta$ and $\alpha \gamma_{\Delta \mathrm{N}}$ heterodimers and the $\alpha_{2} \beta \gamma_{\Delta \mathrm{N}}$ heterotetramer at the low concentration, and an average molecular weight of about $125 \mathrm{kDa}$ and thus appears to be a heterotetramer at the high concentration (Fig. S4B and Table S3). These results indicate that deletion of the N-terminus of the $\gamma$ subunit does not affect the formation of the $\alpha \gamma$ heterodimer, but disrupts the formation of the heterooctamer, which are in agreement with the structural data showing that the $\mathrm{N}$-terminus of the $\gamma$ subunit is not involved in the formation of the $\alpha \gamma$ heterodimer but is critical in the formation of the heterooctamer. The biochemical data also suggest that the $\alpha_{2} \beta \gamma$ heterotetramer appears to be unstable at low concentrations, and the formation of the heterooctamer stabilizes the formation of the $\alpha_{2} \beta \gamma$ heterotetramer.

Consistently, the enzymatic activity assays show that the mutant $\alpha \gamma_{\Delta \mathrm{N}}$ heterodimer exhibits similar enzymatic properties as the wild-type $\alpha \gamma$ heterodimer with comparable $V_{\max }$, $S_{0.5}$, and activation effect (Fig. 4D and Table 2). However, compared to the wild-type 
holoenzyme, the mutant $\alpha_{2} \beta \gamma_{\Delta \mathrm{N}}$ holoenzyme exhibits a significantly low activity in both absence and presence of the activators and displays a very weak activation effect (1.9 folds) (Fig. 4D and Table 2). Specifically, the mutant $\alpha_{2} \beta \gamma_{\Delta \mathrm{N}}$ holoenzyme exhibits a $V_{\max }$ of 5.50 $\mu \mathrm{mol} / \mathrm{mg} / \mathrm{min}$ and a $S_{0.5}$ of $3.85 \mathrm{mM}$ in the absence of the activators, and a $V_{\max }$ of 13.0 $\mu \mathrm{mol} / \mathrm{mg} / \mathrm{min}$ and a $S_{0.5}$ of $2.02 \mathrm{mM}$ in the presence of the activators, which appear to be the averages of those of the $\alpha \beta$ and $\alpha \gamma$ heterodimers. This could be explained as follows: at the enzymatic assay conditions, the mutant $\alpha_{2} \beta \gamma_{\Delta \mathrm{N}}$ heterotetramer has a very low concentration and thus exists mainly as a mixture of the $\alpha \beta$ and $\alpha \gamma_{\Delta \mathrm{N}}$ heterodimers. Taken together, our biochemical data demonstrate that the N-terminal of the $\gamma$ subunit plays an important role in the assembly and function of the holoenzyme.

\section{Discussion}

Human NAD-IDH or IDH3 is a key enzyme in the TCA cycle, which catalyzes the decarboxylation of isocitrate into $\alpha$-ketoglutarate. It exists and functions as a heterooctamer composed of the $\alpha \beta$ and $\alpha \gamma$ heterodimers, and is regulated allosterically and/or competitively by a number of metabolites including CIT, ADP, ATP, and NADH. Our previous biochemical studies of the $\alpha \beta$ and $\alpha \gamma$ heterodimers and the holoenzyme of human IDH3 showed that in the IDH3 holoenzyme, the $\alpha$ subunits of both $\alpha \beta$ and $\alpha \gamma$ heterodimers have catalytic function; but only the $\gamma$ subunit plays a regulatory role, while the $\beta$ subunit plays solely a structural role (Ma et al., 2017b). Our detailed structural and biochemical studies of the isolated $\alpha \gamma$ and $\alpha \beta$ heterodimers revealed the underlying molecular mechanisms (Liu et al., 2018; Ma et al., 2017a; Sun et al., 2020; Sun et al., 2019). Specifically, the $\alpha \gamma$ heterodimer contains an allosteric site in the $\gamma$ subunit which can bind both CIT and ADP. The binding of CIT and ADP induces conformational changes at the allosteric site, which are transmitted to the active site via the heterodimer interface. This series of conformational changes renders the active site to assume an active conformation favorable for ICT binding, leading to the decrease of $S_{0.5, \text { ICT }}$ and hence the activation of the enzyme. In contrast, the $\alpha \beta$ heterodimer contains a pseudo allosteric site in the $\beta$ subunit which is structurally different from the allosteric site and hence cannot bind the activators.

To investigate the molecular mechanism for the assembly and allosteric regulation of the IDH3 holoenzyme, in this work, we determined the crystal structure of human IDH3 holoenzyme in apo form. In the holoenzyme, the $\alpha \beta$ and $\alpha \gamma$ heterodimers form the $\alpha_{2} \beta \gamma$ heterotetramer via their clasp domains, and two $\alpha_{2} \beta \gamma$ heterotetramers assemble the $\left(\alpha_{2} \beta \gamma\right)_{2}$ heterooctamer through the insertion of the N-terminus of the $\gamma$ subunit of one heterotetramer into the back cleft of the $\beta$ subunit of the other heterotetramer. The holoenzyme has a 
438 distorted tetrahedron architecture instead of an architecture with a pseudo 222 symmetry.

439 Specifically, the two $\beta$ and two $\gamma$ subunits are arranged alternately to form the inner core, and

440 the four $\alpha$ subunits are positioned on the periphery. The functional roles of the key residues at

441 the allosteric site, the pseudo allosteric site, the heterodimer interface, and the

442 heterodimer-heterodimer interface, as well as the N-terminus of the $\gamma$ subunit in the assembly

443 and allosteric regulation of the holoenzyme are validated by mutagenesis and kinetic data.

444 The biochemical and structural data also demonstrate that the $\alpha_{2} \beta \gamma$ heterotetramer is unstable

445 because the heterodimer-heterodimer interface is not very tight and involves mainly

446 hydrophobic interactions. On the other hand, the $\left(\alpha_{2} \beta \gamma\right)_{2}$ heterooctamer is very stable as the

447 two $\alpha_{2} \beta \gamma$ heterotetramers interact with each other via two large interfaces to form a ring-like

448 architecture, and the interfaces involve both hydrophilic and hydrophobic interactions. The

449 formation of the $\left(\alpha_{2} \beta \gamma\right)_{2}$ heterooctamer stabilizes the formation of the $\alpha_{2} \beta \gamma$ heterotetramer in

450 the holoenzyme. These findings reveal the molecular mechanism for the assembly of the

451 heterotetramer and heterooctamer of human IDH3.

452 Structural comparison shows that in the holoenzyme, the $\alpha \gamma$ heterodimer assumes very

453 similar overall conformation as the isolated $\alpha^{\mathrm{Mg}} \gamma$ heterodimer, and the allosteric site assumes

454 a proper conformation to bind the activators. However, the $\alpha \beta$ heterodimer exhibits some

455 conformational changes from the isolated $\alpha^{\mathrm{Ca}} \beta$ heterodimer. The formation of the $\left(\alpha_{2} \beta \gamma\right)_{2}$

456 heterooctamer renders the $\alpha \beta$ heterodimer to adopt an overall conformation similar to that of

457 the $\alpha^{\mathrm{Mg}} \gamma$ heterodimer rather than the compact conformation of the $\alpha^{\mathrm{Ca}} \beta$ heterodimer.

458 Nevertheless, the pseudo allosteric site is still unable to bind the activators. Hence, the $\alpha$

459 subunit of the $\alpha \beta$ heterodimer in the holoenzyme can be allosterically activated and has

460 normal catalytic function but the $\beta$ subunit still has no regulatory function. These results also

461 demonstrate that the structure characteristics and the regulatory mechanisms of the $\alpha \beta$ and $\alpha \gamma$

462 heterodimers uncovered from the structure and biochemical studies of the isolated $\alpha \beta$ and $\alpha \gamma$

463 heterodimers are largely applicable to the holoenzyme.

464 Our biochemical and structural data show that the IDH3 holoenzyme exists as a stable 465 heterooctamer in both solution and structure, and functions as a heterooctamer as well. The 466 wild-type holoenzyme exhibits a notably higher activity than the sum of the activities of the $467 \alpha \beta$ and $\alpha \gamma$ heterodimers in both the absence and presence of activators, and that the mutant 468 holoenzyme containing the $\alpha_{\mathrm{Y} 126 \mathrm{~A}}$ mutation at the active site in either the $\alpha \beta$ or $\alpha \gamma$ 469 heterodimer exhibits about $50 \%$ of the activity of the wild-type holoenzyme and displays a 470 significant activation effect; however, the mutant holoenzyme containing the $\alpha_{Y 126 \mathrm{~A}}$ mutation 471 in both $\alpha \beta$ and $\alpha \gamma$ heterodimers completely abolishes the activity (Ma et al., 2017b). These 472 results indicate that in the holoenzyme, both $\alpha \beta$ or $\alpha \gamma$ heterodimer have catalytic function and 
473 can be activated by the activators, and the binding of the activators to the allosteric site in the

$474 \gamma$ subunit can allosterically regulate the $\alpha$ subunit in both heterodimers. The structure of the

475 IDH3 holoenzyme shows that the allosteric site in the $\gamma$ subunit could bind the activators but 476 the pseudo allosteric site in the $\beta$ subunit remains incapable of binding the activators, and that 477 the overall conformation and the active-site conformation in both $\alpha \beta$ and $\alpha \gamma$ heterodimers are 478 suitable for allosteric activation and catalytic function. Consistently, the biochemical data 479 show that the mutations at the allosteric site have significant impacts on the activation and 480 function of the holoenzyme, whereas the mutations at the pseudo allosteric site have 481 insignificant impacts on the activation and function of the holoenzyme, indicating that the 482 allosteric site plays a critical role and the pseudo allosteric site has no regulatory role in the 483 allosteric activation of the holoenzyme. In addition, the mutations at the heterodimer 484 interfaces have severe impacts on the activation and function of the holoenzyme, indicating 485 that the heterodimer interfaces play a vital role in the communication from the allosteric site 486 to the active sites of both $\alpha$ subunits. Furthermore, while the $\beta_{\mathrm{E} 150 \mathrm{~A}}$ and $\gamma_{\mathrm{E} 148 \mathrm{~A}}$ mutations at the 487 heterodimer-heterodimer interface have minor impacts on the activation and function of the 488 holoenzyme; the $\alpha_{\mathrm{Q} 139 \mathrm{~A}}$ mutation in either or both the $\alpha \beta$ and $\alpha \gamma$ heterodimers renders the 489 mutant holoenzyme constitutively active in both the absence and presence of the activators, 490 indicating that the heterodimer-heterodimer interface plays an important role in the assembly 491 and allosteric regulation of the $\alpha_{2} \beta \gamma$ heterotetramer and the holoenzyme. Taken together, the 492 structural and biochemical data suggest that upon the binding of the activators to the allosteric 493 site, the activation signal is transmitted from the allosteric site to the $\alpha$ subunits of both $\alpha \beta$ 494 and $\alpha \gamma$ heterodimers through the heterodimer and heterodimer-heterodimer interfaces, leading 495 to the activation of both heterodimers in the $\alpha_{2} \beta \gamma$ heterotetramer and the holoenzyme. These findings reveal the molecular mechanism for the allosteric regulation of the IDH3 holoenzyme.

All eukaryotes contain NAD-IDHs to carry out the catalytic function in the TCA cycle. However, the composition of NAD-IDHs differs from low eukaryotes to high eukaryotes. In low eukaryotes such as Saccharomyces cerevisiae and most single cell eukaryotes, the NAD-IDH is composed of two types of subunits (IDH1 and IDH2) in 1:1 ratio. IDH1 and IDH2 form the IDH1/IDH2 heterodimer which assembles the heterotetramer and further the heterooctamer. In high eukaryotes such as mammals, the NAD-IDH is composed of three types of subunits $(\alpha, \beta$ and $\gamma)$ in 2:1:1 ratio. The $\alpha, \beta$ and $\gamma$ subunits form two types of heterodimers ( $\alpha \beta$ and $\alpha \gamma)$ which assemble the $\alpha_{2} \beta \gamma$ heterotetramer and further the $\left(\alpha_{2} \beta \gamma\right)_{2}$ heterooctamer. In either cases, the NAD-IDHs always exist and function as the heterooctamer.

Previous biochemical and structural studies showed that in yeast NAD-IDH, IDH2 is the catalytic subunit which contains the active site, and IDH1 is the regulatory subunit which 
509 contains the allosteric site (Cupp and Mcalisterhenn, 1993; Lin and McAlister-Henn, 2002,

510 2003). The heterooctamer of yeast NAD-IDH exhibits an asymmetric architecture, in which

511 the regulatory IDH1 subunits form the inner core and the catalytic IDH2 subunits are

512 positioned on the outside surface, and thus the four IDH1 subunits are in two different

513 structural environments with different conformations (Taylor et al., 2008).

514 Although early biochemical studies of mammalian NAD-IDHs showed that the $\alpha$ subunit

515 is the catalytic subunit and the $\beta$ and $\gamma$ subunits are the regulatory subunits, our biochemical

516 and structural studies of human NAD-IDH or IDH3 clearly demonstrated that the $\alpha$ subunits

517 of both $\alpha \beta$ and $\alpha \gamma$ heterodimers have the catalytic function, the $\gamma$ subunit plays the regulatory

518 role, whereas the $\beta$ subunit plays no regulatory role albeit it is required for the function of the

519 holoenzyme (Ma et al., 2017b; Sun et al., 2019). Interestingly, the heterooctamer of human

520 NAD-IDH also exhibits an asymmetric architecture, in which the two $\beta$ subunits and two $\gamma$

521 subunits are arranged alternately to form the inner core and the four $\alpha$ subunits are positioned

522 on the outer surface, and the two $\beta$ subunits are in different structural environments with

523 different conformations from the two $\gamma$ subunits. Structural comparison shows that the

524 heterooctamers of yeast and human NAD-IDHs exhibit almost identical architecture and

525 could be superimposed very well. These results suggest that like human NAD-IDH, only two

526 of the four IDH1 subunits in yeast NAD-IDH have allosteric regulatory function and the other

527 two have no regulatory function. This explains the biochemical data that there are only two

528 AMP-binding sites in yeast NAD-IDH holoenzyme, and provides the support evidence for the

529 speculation that the binding of AMP to all four IDH1 subunits is an artifact of excess AMP in

530 the crystallization solution (Cupp and Mcalisterhenn, 1993; Lin and McAlister-Henn, 2002,

531 2003; McAlister-Henn, 2012; Taylor et al., 2008; Zhao and McAlister-Henn, 1997). These

532 findings also suggest that all eukaryotic NAD-IDHs would assume a similar asymmetric

533 architecture and employ a similar allosteric regulation mechanism. 


\section{Cloning, expression and purification}

537 The $\alpha \beta$ and $\alpha \gamma$ heterodimers and the holoenzyme of human IDH3 were prepared as described 538 previously (Ma et al., 2017b). Briefly, the DNA fragments encoding the $\alpha, \beta$ and $\gamma$ subunits of 539 human IDH3 were cloned into the co-expression vector pQlinkN with the C-terminals of the $\beta$ 540 and $\gamma$ subunits attached with a TEV protease cleavage site and a His ${ }_{6}$ tag to construct the

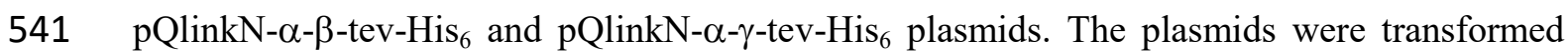
542 into E. coli BL21 (DE3) Codon-Plus strain (Novagen). When the culture of the transformed 543 cells reached an $\mathrm{OD}_{600}$ of 0.5 , the protein expression was induced by $0.4 \mathrm{mM}$ IPTG for $20 \mathrm{hrs}$ 544 at $24{ }^{\circ} \mathrm{C}$. The bacterial cells were harvested and then sonicated on ice in the lysis buffer (50 $545 \mathrm{mM}$ HEPES, pH 7.4, $200 \mathrm{mM} \mathrm{NaCl}, 0.2 \mathrm{mM} \mathrm{MnCl}_{2}, 10 \%$ glycerol, and $7.2 \mathrm{mM} \beta$-ME) 546 supplemented with $1 \mathrm{mM}$ PMSF. The target proteins were purified by affinity 547 chromatography using a Ni-NTA column (Qiagen) with the lysis buffer supplemented with 20 $548 \mathrm{mM}$ and $200 \mathrm{mM}$ imidazole serving as the washing buffer and elution buffer, respectively. 549 The elution fraction was dialyzed overnight against the lysis buffer supplemented with TEV 550 protease to cleave the $\mathrm{His}_{6}$-tag of the target protein. The cleavage mixture was reloaded on a 551 Ni-NTA column and washed with the lysis buffer supplemented with $10 \mathrm{mM}$ imidazole. The

552 flow-through fraction containing the target protein was further purified by gel filtration using 553 a Superdex 200 10/60 GL column (GE Healthcare) equilibrated with the storage buffer (10 $554 \mathrm{mM}$ HEPES, pH 7.4, $200 \mathrm{mM} \mathrm{NaCl}$, and $5 \mathrm{mM} \beta$-ME). The holoenzyme was prepared by 555 co-purifying the separately expressed $\alpha \beta$ and $\alpha \gamma$ heterodimers using the same methods as for 556 the $\alpha \beta$ and $\alpha \gamma$ heterodimers. Purity of the proteins was analyzed by $12 \%$ SDS-PAGE with 557 Coomassie blue staining. Mutants of the $\alpha \beta$ and $\alpha \gamma$ heterodimers and the holoenzyme 558 containing point mutations were constructed using the QuikChange ${ }^{\circledR}$ Site-Directed

559 Mutagenesis kit (Strategene). Expression and purification of the mutants were carried out 560 using the same methods as for the wild-type proteins.

\section{SEC-MALS analysis}

563 The purity, molecular mass, and size distribution of the proteins were analyzed by an 564 analytical light scattering instrument (SEC-MALS) consisting of an Agilent 1260 Infinity 565 Isocratic Liquid Chromatography System, a Wyatt Dawn Heleos II Multi-Angle Light 566 Scattering Detector, and a Wyatt Optilab T-rEX Refractive Index Detector (Wyatt 567 Technology). Analytical size exclusion chromatography was performed at $24{ }^{\circ} \mathrm{C}$ using a 568 Superdex 200 10/300 GL column (GE Healthcare) equilibrated with a mobile phase 569 containing $10 \mathrm{mM}$ HEPES (pH 7.4), $200 \mathrm{mM} \mathrm{NaCl}$, and $5 \mathrm{mM} \beta$-ME. $100 \mu$ protein solution 570 was injected into the column and eluted at a flow rate of $0.4 \mathrm{ml} / \mathrm{min}$. The column effluent was 
571 monitored simultaneously with three detectors for UV absorption, light scattering and

572 refractive index. The data were analyzed using the ASTRA software (Wyatt Technology) to

573 determine the molecular mass of the protein (Folta-Stogniew, 2006).

575 Crystallization, diffraction data collection and structure determination

576 Crystallization was performed using the hanging drop vapor diffusion method at $20{ }^{\circ} \mathrm{C}$ by

577 mixing equal volume of protein solution $(10 \mathrm{mg} / \mathrm{ml})$ and reservoir solution. Crystals of the

578 IDH3 holoenzyme grew in drops containing the reservoir solution of $0.05 \mathrm{M} \mathrm{NH}_{4} \mathrm{Cl}, 0.05$

579 Bis-Tris ( $\mathrm{pH}$ 6.5), and 30\% pentaerythritol ethoxylate. Crystals were cryoprotected using the 580 reservoir solution supplemented with $25 \%$ ethylene glycol. Diffraction data were collected at

$581100 \mathrm{~K}$ at BL17U1 of Shanghai Synchrotron Radiation Facility and processed with HKL3000

582 (Otwinowski Z., 1997). Statistics of the diffraction data are summarized in Table 1.

583 The structure of the IDH3 holoenzyme was solved with the molecular replacement 584 method implemented in program Phaser (McCoy et al., 2007) using the structures of the $\alpha \gamma$ 585 heterodimer (PDB code 6KDE) and the $\alpha \beta$ heterodimer (PDB code 5GRH) as the search 586 models. Structure refinement was carried out with program Phenix and REFMAC5 (Adams et 587 al., 2010; Murshudov et al., 1997). Model building was performed with program Coot 588 (Emsley and Cowtan, 2004). Stereochemistry and quality of the structure model were 589 analyzed using programs in the CCP4 suite (Winn et al., 2011). Structure figures were 590 prepared using PyMol (Schrodinger, 2010). Statistics of the structure refinement and the final 591 structure model are also summarized in Table 1.

\section{Enzymatic activity assay}

594 The enzymatic activities of the wild-type and mutant $\alpha \beta$ and $\alpha \gamma$ heterodimers and 595 holoenzymes of human IDH3 were determined using the method as described previously (Ma 596 et al., 2017b). The standard reaction solution $(1 \mathrm{ml})$ consisted of $2 \mathrm{ng} / \mathrm{ml}$ enzyme, $33 \mathrm{mM}$ 597 Tris-acetate ( $\mathrm{pH}$ 7.4), $40 \mathrm{mM}$ ICT, $2 \mathrm{mM} \mathrm{Mn}^{2+}$, and $3.2 \mathrm{mM} \mathrm{NAD}$. The activity is defined as 598 the moles of NADH produced per min per milligram of enzyme $(\mathrm{mol} / \mathrm{min} / \mathrm{mg})$. The kinetic 599 data in the absence of the activators (CIT and ADP) were measured with varied 600 concentrations of ICT $(0-40 \mathrm{mM}), \mathrm{Mn}^{2+}(0-10 \mathrm{mM})$, or NAD $(0-10 \mathrm{mM})$ to obtain the $\mathrm{V}_{\max }$ 601 and $\mathrm{S}_{0.5}$ for ICT, $\mathrm{Mn}^{2+}$, or NAD, respectively. The kinetic data in the presence of the activators 602 were measured at the same conditions supplemented with $1 \mathrm{mM}$ CIT and $1 \mathrm{mM}$ ADP. The 603 kinetic parameters were obtained by fitting the kinetic data into the non-Michaelis-Menten 604 equation "V=Vmax*[S $]^{\wedge} \mathrm{h} /\left(\mathrm{S}_{0.5} \wedge \mathrm{h}+[\mathrm{S}]^{\wedge} \mathrm{h}\right)$ " using program Graphpad Prism (Graphpad 605 Software). All experiments were performed twice and the values were the averages of the 606 measurements with the standard errors. 


\section{Protein Data Bank accession code}

608 The crystal structure of human IDH3 holoenzyme has been deposited in the Protein Data

609 Bank with accession code 7CE3.

610

\section{Conflict of interest statement}

612 The authors declare no conflict of interests.

613

614 Acknowledgements

615 We thank the staff members at BL17U1 of Shanghai Synchrotron Radiation Facility (SSRF)

616 and the Large-scale Protein Preparation System at the National Facility for Protein Science in

617 Shanghai (NFPS), Zhangjiang Lab, China for providing technical support and assistance in

618 data collection and analysis, and other members of our group for valuable discussion. This

619 work was supported by grants from the National Natural Science Foundation of China

620 (31870723 and 31530013) and the CAS Facility-based Open Research Program.

621

622 Authorship contributions

623 PS carried out the biochemical and structural studies, and participated in the data analyses.

624 TM participated in the initial biochemical and structural studies. JD conceived the study,

625 participated in the experimental design and data analyses, and wrote the manuscript.

626 


\section{References}

Adams, P.D., Afonine, P.V., Bunkoczi, G., Chen, V.B., Davis, I.W., Echols, N., Headd, J.J., Hung, L.W., Kapral, G.J., Grosse-Kunstleve, R.W., McCoy, A.J., Moriarty, N.W., Oeffner, R., Read, R.J., Richardson, D.C., Richardson, J. S., Terwilliger, T. C., Zwart, P. H. (2010). PHENIX: a comprehensive Python-based system for macromolecular structure solution. Acta Crystallogr. D Biol. Crystallogr. 66, 213-221. DOI: https://doi.org/10.1107/S0907444909052925

Al-Khallaf, H. (2017). Isocitrate dehydrogenases in physiology and cancer: biochemical and molecular insight. Cell Biosci. 7, 37. DOI: https://doi.org/10.1186/s13578-017-0165-3

Cohen, P.F., and Colman, R.F. (1972). Diphosphopyridine nucleotide dependent isocitrate dehydrogenase from pig heart. Charactgerization of the active substrate and modes of regulation. Biochemistry 11, 1501-1508. DOI: https://doi.org/10.1021/bi00758a027

Cupp, J.R., and Mcalisterhenn, L. (1993). Kinetic-analysis of NAD ${ }^{+}$-isocitrate dehydrogenase with altered isocitrate binding-sites-contribution of Idh1 and Idh2 subunits to regulation and catalysis. Biochemistry 32, 9323-9328. DOI: https://doi.org/10.1021/bi00087a010

Dang, L., White, D.W., Gross, S., Bennett, B.D., Bittinger, M.A., Driggers, E.M., Fantin, V.R., Jang, H.G., Jin, S., Keenan, M.C., Marks, K. M., Prins, R. M., Ward, P. S., Yen, K. E., Liau, L. M., Rabinowitz, J. D., Cantley, L. C., Thompson, C. B., Heiden, M. G. V., Su, S. M. (2009). Cancer-associated IDH1 mutations produce 2-hydroxyglutarate. Nature 462, 739-U752. DOI: https://doi.org/10.1038/nature08617

Ehrlich, R.S., and Colman, R. (1983). Separation, recombination, and characterization of dissimilar subunits of the DPN-dependent isocitrate dehydrogenase from pig heart. J. Biol. Chem. 258, 7079-7086.

Ehrlich, R.S., and Colman, R.F. (1981). Binding of ligands to half of subunits of NAD-dependent isocitrate dehydrogenase from pig-heart-binding of manganous ion, isocitrate, ADP and NAD. J. Biol. Chem. 256, 1276-1282.

Ehrlich, R.S., Hayman, S., Ramachandran, N., and Colman, R. (1981). Re-evaluation of molecular weight of pig heart NAD-specific isocitrate dehydrogenase. J. Biol. Chem. 256, 10560-10564.

Emsley, P., and Cowtan, K. (2004). Coot: model-building tools for molecular graphics. Acta Crystallogr. D Biol. Crystallogr. 60, 2126-2132. DOI: https://doi.org/10.1107/S0907444904019158

Folta-Stogniew, E. (2006). Oligomeric states of proteins determined by size-exclusion chromatography coupled with light scattering, absorbance, and refractive index detectors. Methods Mol. Biol. 328, 97-112. DOI: https://doi.org/10.1385/1-59745-026-X:97

Gabriel, J., and Plaut, G. (1984a). Citrate activation of NAD-specific isocitrate dehydrogenase from bovine heart. J. Biol. Chem. 259, 1622-1628.

Gabriel, J.L., Milner, R., and Plaut, G.W. (1985). Inhibition and activation of bovine heart NAD-specific isocitrate dehydrogenase by ATP. Arch. Biochem. Biophys. 240, 128-134. DOI: https://doi.org/10.1016/0003-9861(85)90015-3

Gabriel, J.L., and Plaut, G.W. (1984b). Inhibition of bovine heart NAD-specific isocitrate dehydrogenase by reduced pyridine nucleotides: modulation of inhibition by $\mathrm{ADP}, \mathrm{NAD}^{+}$, $\mathrm{Ca}^{2+}$, citrate, and isocitrate. Biochemistry 23, 2773-2778. DOI: https://doi.org/10.1021/bi00307a037

Hartong, D.T., Dange, M., McGee, T.L., Berson, E.L., Dryja, T.P., and Colman, R.F. (2008). Insights from retinitis pigmentosa into the roles of isocitrate dehydrogenases in the Krebs cycle. Nat Genet. 40, 1230-1234. DOI: https://doi.org/10.1038/ng.223

Hurley, J.H., Dean, A.M., Koshland, D.E., Jr., and Stroud, R.M. (1991). Catalytic mechanism of $\mathrm{NADP}^{+}$-dependent isocitrate dehydrogenase: implications from the structures of magnesium-isocitrate and $\mathrm{NADP}^{+}$complexes. Biochemistry 30, 8671-8678. DOI: https://doi.org/10.1021/bi00099a026

Jo, S.H., Son, M.K., Koh, H.J., Lee, S.M., Song, I.H., Kim, Y.O., Lee, Y.S., Jeong, K.S., Kim, W.B., Park, J.W., Song, B. J., Huh, T. L. (2001). Control of mitochondrial redox balance and cellular defense against oxidative damage by mitochondrial $\mathrm{NADP}^{+}$-dependent 
isocitrate dehydrogenase. J. Biol. Chem. 276, 16168-16176. DOI: https://doi.org/10.1074/jbc.M010120200

Kiefmann, M., Tank, S., Keller, P., Bornchen, C., Rinnenthal, J.L., Tritt, M.O., Schulte-Uentrop, L., Olotu, C., Goetz, A.E., and Kiefmann, R. (2017). IDH3 mediates apoptosis of alveolar epithelial cells type 2 due to mitochondrial $\mathrm{Ca}^{2+}$ uptake during hypocapnia. Cell Death Dis. 8, e3005. DOI: https://doi.org/10.1038/cddis.2017.403

Kim, S.Y., and Park, J.W. (2003). Cellular defense against singlet oxygen-induced oxidative damage by cytosolic $\mathrm{NADP}^{+}$-dependent isocitrate dehydrogenase. Free Radic. Res. 37, 309-316. DOI: https://doi.org/10.1080/1071576021000050429

Koh, H.J., Lee, S.M., Son, B.G., Lee, S.H., Ryoo, Z.Y., Chang, K.T., Park, J.W., Park, D.C., Song, B.J., Veech, R.L., Song, H., Huh, T. L. (2004). Cytosolic NADP ${ }^{+}$-dependent isocitrate dehydrogenase plays a key role in lipid metabolism. J. Biol. Chem. 279, 39968-39974. DOI: https://doi.org/10.1074/jbc.M402260200

Lee, S.M., Koh, H.J., Park, D.C., Song, B.J., Huh, T.L., and Park, J.W. (2002). Cytosolic $\mathrm{NADP}^{+}$-dependent isocitrate dehydrogenase status modulates oxidative damage to cells. Free Radic. Biol. Med. 32, 1185-1196. DOI: https://doi.org/10.1016/s0891-5849(02)00815-8

Lin, A.P., Demeler, B., Minard, K.I., Anderson, S.L., Schirf, V., Galaleldeen, A., and McAlister-Henn, L. (2011). Construction and analyses of tetrameric forms of yeast $\mathrm{NAD}^{+}$-specific isocitrate dehydrogenase. Biochemistry 50, 230-239. DOI: https://doi.org/10.1021/bi101401h

Lin, A.P., and McAlister-Henn, L. (2002). Isocitrate binding at two functionally distinct sites in yeast $\mathrm{NAD}^{+}$-specific isocitrate dehydrogenase. J. Biol. Chem. 277, 22475-22483. DOI: https://doi.org/10.1074/jbc.M202534200

Lin, A.P., and McAlister-Henn, L. (2003). Homologous binding sites in yeast isocitrate dehydrogenase for cofactor $\left(\mathrm{NAD}^{+}\right.$) and allosteric activator (AMP). J. Biol. Chem. 278, 12864-12872. DOI: https://doi.org/10.1074/jbc.M300154200

Liu, Y., Hu, L., Ma, T., Yang, J., and Ding, J. (2018). Insights into the inhibitory mechanisms of NADH on the $\alpha \gamma$ heterodimer of human NAD-dependent isocitrate dehydrogenase. Sci. Rep. 8, 3146. DOI: https://doi.org/10.1038/s41598-018-21584-7

Ma, T., Peng, Y., Huang, W., and Ding, J. (2017a). Molecular mechanism of the allosteric regulation of the $\alpha \gamma$ heterodimer of human NAD-dependent isocitrate dehydrogenase. Sci. Rep. 7, 40921. DOI: https://doi.org/10.1038/srep40921

Ma, T., Peng, Y., Huang, W., Liu, Y., and Ding, J. (2017b). The $\beta$ and $\gamma$ subunits play distinct functional roles in the $\alpha_{2} \beta \gamma$ heterotetramer of human NAD-dependent isocitrate dehydrogenase. Sci. Rep. 7, 41882. DOI: https://doi.org/10.1038/srep41882

May, J.L., Kouri, F.M., Hurley, L.A., Liu, J., Tommasini-Ghelfi, S., Ji, Y.R., Gao, P., Calvert, A.E., Lee, A., Chandel, N.S., Davuluri, R. V., Horbinski, C. M., Locasale, J. W., Stegh, A. H. (2019). IDH3 $\alpha$ regulates one-carbon metabolism in glioblastoma. Sci. Adv. 5. DOI: https://doi.org/10.1126/sciadv.aat0456

McAlister-Henn, L. (2012). Ligand binding and structural changes associated with allostery in yeast $\mathrm{NAD}^{+}$-specific isocitrate dehydrogenase. Arch. Biochem. Biophys. 519, 112-117. DOI: https://doi.org/10.1016/j.abb.2011.10.003

McCoy, A.J., Grosse-Kunstleve, R.W., Adams, P.D., Winn, M.D., Storoni, L.C., and Read, R.J. (2007). Phaser crystallographic software. J. Appl. Crystallogr. 40, 658-674. DOI: https://doi.org/10.1107/S0021889807021206

Murshudov, G.N., Vagin, A.A., and Dodson, E.J. (1997). Refinement of macromolecular structures by the maximum-likelihood method. Acta Crystallogr. D Biol. Crystallogr. 53, 240-255. DOI: https://doi.org/10.1107/S0907444996012255

Nichols, B.J., Hall, L., Perry, A.C., and Denton, R.M. (1993). Molecular cloning and deduced amino acid sequences of the $\gamma$ subunits of rat and monkey $\mathrm{NAD}^{+}$-isocitrate dehydrogenases. Biochem. J. 295, 347-350.

Nichols, B.J., Perry, A.C., Hall, L., and Denton, R.M. (1995). Molecular cloning and deduced amino acid sequences of the $\alpha$ and $\beta$ subunits of mammalian $\mathrm{NAD}^{+}$-isocitrate 
dehydrogenase. Biochem. J. 310, 917-922.

Otwinowski Z., M.W. (1997). Processing of X-ray diffraction data collected in oscillation mode. Methods in Enzymol. 276, 307-326.

Pavlova, N.N., and Thompson, C.B. (2016). The emerging hallmarks of cancer metabolism. Cell Metab. 23, 27-47. DOI: https://doi.org/10.1016/j.cmet.2015.12.006

Schrodinger, LLC (2010). The PyMOL molecular graphics system, Version 1.3r1.

Sun, P., Bai, T., Ma, T., and Ding, J. (2020). Molecular mechanism of the dual regulatory roles of ATP on the $\alpha \gamma$ heterodimer of human NAD-dependent isocitrate dehydrogenase. Sci. Rep. 10, 6225-6225. DOI: https://doi.org/10.1038/s41598-020-63425-6

Sun, P., Ma, T., Zhang, T., Zhu, H., Zhang, J., Liu, Y., and Ding, J. (2019). Molecular basis for the function of the $\alpha \beta$ heterodimer of human NAD-dependent isocitrate dehydrogenase. J. Biol. Chem. 294, 16214-16227. DOI: https://doi.org/10.1074/jbc.RA119.010099

Taylor, A.B., Hu, G., Hart, P.J., and McAlister-Henn, L. (2008). Allosteric motions in structures of yeast $\mathrm{NAD}^{+}$-specific isocitrate dehydrogenase. J. Biol. Chem. 283, 10872-10880. DOI: https://doi.org/10.1074/jbc.M708719200

Waitkus, M.S., Diplas, B.H., and Yan, H. (2016). Isocitrate dehydrogenase mutations in gliomas. Neuro Oncol. 18, 16-26. DOI: https://doi.org/10.1093/neuonc/nov136

Winn, M.D., Ballard, C.C., Cowtan, K.D., Dodson, E.J., Emsley, P., Evans, P.R., Keegan, R.M., Krissinel, E.B., Leslie, A.G., McCoy, A., McNicholas, S. J., Murshudov, G. N., Pannu, N. S., Potterton, E. A., Powell, H. R., Read, R. J., Vagin, A., Wilson, K. S. (2011). Overview of the CCP4 suite and current developments. Acta Crystallogr. D Biol. Crystallogr. 67, 235-242. DOI: https://doi.org/10.1107/S0907444910045749

Xu, X., Zhao, J., Xu, Z., Peng, B., Huang, Q., Arnold, E., and Ding, J. (2004). Structures of human cytosolic NADP-dependent isocitrate dehydrogenase reveal a novel self-regulatory mechanism of activity. J. Biol. Chem. 279, 33946-33957. DOI: https://doi.org/10.1074/jbc.M404298200

Yan, H., Parsons, D.W., Jin, G., McLendon, R., Rasheed, B.A., Yuan, W., Kos, I., Batinic-Haberle, I., Jones, S., Riggins, G.J., Friedman, H., Friedman, A., Reardon, D., Herndon, J., Kinzler, K. W., Velculescu, V. E., Vogelstein, B., Bigner, D. D. (2009). IDH1 and IDH2 mutations in gliomas. N. Engl. J. Med. 360, 765-773. DOI: https://doi.org/10.1056/NEJMoa0808710

Yang, B., Zhong, C., Peng, Y., Lai, Z., and Ding, J. (2010). Molecular mechanisms of "off-on switch" of activities of human IDH1 by tumor-associated mutation R132H. Cell Res. 20, 1188-1200. DOI: https://doi.org/10.1038/cr.2010.145

Yoshimi, N., Futamura, T., Bergen, S.E., Iwayama, Y., Ishima, T., Sellgren, C., Ekman, C.J., Jakobsson, J., Palsson, E., Kakumoto, K., Ohgi, Y., Yoshikawa, T., Lande 'n, M., Hashimoto, K. (2016). Cerebrospinal fluid metabolomics identifies a key role of isocitrate dehydrogenase in bipolar disorder: evidence in support of mitochondrial dysfunction hypothesis. Mol. Psychiatry 21, 1504-1510. DOI: https://doi.org/10.1038/mp.2015.217

Zhang, D.X., Wang, Y.B., Shi, Z.M., Liu, J.Y., Sun, P., Hou, X.D., Zhang, J., Zhao, S.M., Zhou, B.H.P., and Mi, J. (2015). Metabolic reprogramming of cancer-associated fibroblasts by IDH3 $\alpha$ downregulation. Cell Rep. 10, 1335-1348. DOI: https://doi.org/10.1016/j.celrep.2015.02.006

Zhao, W.N., and McAlister-Henn, L. (1997). Affinity purification and kinetic analysis of mutant forms of yeast $\mathrm{NAD}^{+}$-specific isocitrate dehydrogenase. J. Biol. Chem. 272, 21811-21817.

Zheng, J., and Jia, Z. (2010). Structure of the bifunctional isocitrate dehydrogenase kinase/phosphatase. Nature 465, 961-965. DOI: https://doi.org/10.1038/nature09088

Zheng, J., Yates, S.P., and Jia, Z. (2012). Structural and mechanistic insights into the bifunctional enzyme isocitrate dehydrogenase kinase/phosphatase AceK. Philos. Trans. R Soc. Lond. B Biol. Sci. 367, 2656-2668. DOI: https://doi.org/10.1098/rstb.2011.0426 